\title{
High-mass Starless Clumps in the Inner Galactic Plane: The Sample and Dust Properties
}

\author{
Jinghua Yuan (袁敬华) ${ }^{1,10}$, \\ Yuefang Wu (吴月芳 $)^{2}$, Simon P. Ellingsen ${ }^{3}$, Neal J. Evans II ${ }^{4,5}$, Christian Henkel ${ }^{6,7}$, \\ Ke Wang (王科 $)^{8}$, Hong-Li Liu (刘洪礼) ${ }^{1}$, Tie Liu (刘铁) $)^{5}$, Jin-Zeng Li (李金增 $)^{1}$, and Annie Zavagno ${ }^{9}$ \\ ${ }^{1}$ National Astronomical Observatories, Chinese Academy of Sciences, 20A Datun Road, Chaoyang District, Beijing 100012, China; jhyuan@nao.cas.cn \\ ${ }^{2}$ Department of Astronomy, Peking University, 100871 Beijing, China \\ ${ }^{3}$ School of Physical Sciences, University of Tasmania, Hobart, Tasmania, Australia \\ ${ }^{4}$ Department of Astronomy, The University of Texas at Austin, 2515 Speedway, Stop C1400, Austin, TX 78712-1205, USA \\ ${ }^{5}$ Korea Astronomy and Space Science Institute 776, Daedeokdae-ro, Yuseong-gu, Daejeon 34055, Korea \\ ${ }^{6}$ Max-Planck-Institut für Radioastronomie, Auf dem Hügel 69, D-53121 Bonn, Germany \\ ${ }^{7}$ Astron. Dept., King Abdulaziz University, P.O. Box 80203, Jeddah 21589, Saudi Arabia \\ ${ }^{8}$ European Southern Observatory, Karl-Schwarzschild-Str. 2, D-85748 Garching bei München, Germany \\ ${ }^{9}$ Aix Marseille Universit, CNRS, LAM (Laboratoire d'Astrophysique de Marseille) UMR 7326, F-13388, Marseille, France \\ Received 2016 December 23; revised 2017 March 30; accepted 2017 May 5; published 2017 July 20
}

\begin{abstract}
We report a sample of 463 high-mass starless clump (HMSC) candidates within $-60^{\circ}<l<60^{\circ}$ and $-1^{\circ}<b<1^{\circ}$. This sample has been singled out from 10,861 ATLASGAL clumps. None of these sources are associated with any known star-forming activities collected in SIMBAD and young stellar objects identified using color-based criteria. We also make sure that the HMSC candidates have neither point sources at 24 and $70 \mu \mathrm{m}$ nor strong extended emission at $24 \mu \mathrm{m}$. Most of the identified HMSCs are infrared dark, and some are even dark at $70 \mu \mathrm{m}$. Their distribution shows crowding in Galactic spiral arms and toward the Galactic center and some wellknown star-forming complexes. Many HMSCs are associated with large-scale filaments. Some basic parameters were attained from column density and dust temperature maps constructed via fitting far-infrared and submillimeter continuum data to modified blackbodies. The HMSC candidates have sizes, masses, and densities similar to clumps associated with Class II methanol masers and H II regions, suggesting that they will evolve into star-forming clumps. More than $90 \%$ of the HMSC candidates have densities above some proposed thresholds for forming highmass stars. With dust temperatures and luminosity-to-mass ratios significantly lower than that for star-forming sources, the HMSC candidates are externally heated and genuinely at very early stages of high-mass star formation. Twenty sources with equivalent radii $r_{\mathrm{eq}}<0.15 \mathrm{pc}$ and mass surface densities $\Sigma>0.08 \mathrm{~g} \mathrm{~cm}^{-2}$ could be possible high-mass starless cores. Further investigations toward these HMSCs would undoubtedly shed light on comprehensively understanding the birth of high-mass stars.
\end{abstract}

Key words: infrared: ISM - ISM: clouds - stars: formation - stars: massive - submillimeter: ISM

Supporting material: figure sets, machine-readable tables

\section{Introduction}

High-mass stars, through mechanical and radiative input, play crucial roles in the structural formation and evolution in galaxies. They are also the primary contributor of chemical enrichment in space. However, the forming process of highmass stars still remains a mystery (Tan et al. 2014).

The turbulent core and competitive accretion models have been proposed as alternative scenarios for the formation of massive stars (Bonnell et al. 2001; McKee \& Tan 2003). In the turbulent core model, the final stellar mass is pre-assembled in the collapsing core, so this model requires the existence of high-mass starless cores. In contrast, the competitive accretion model predicts that high-mass stars begin as clusters of small cores with masses peaked around the Jeans mass of the clump and there is no connection between the mass of its birth core and the final stellar mass. Discriminating between these two models requires identification of the youngest high-mass star formation regions to enable investigation of the initial conditions.

\footnotetext{
${ }^{10}$ FITS images for the far-IR to submillimeter data, $\mathrm{H}_{2}$ column density, and dust temperature maps of all the HMSC candidates are available at https:// yuanjinghua.github.io/hmscs.html. Codes used for this work are publicly available from https://github.com/yuanjinghua/HMSCs_cat.
}

Starless clumps are the objects that fragment into dense starless cores $(r<0.15 \mathrm{pc})$, which subsequently contract to form individual or bound systems of protostars (Tan et al. 2014). Despite many searches, there are only a few candidate high-mass starless cores known to date (e.g., Wang et al. 2011, 2014; Tan et al. 2013; Cyganowski et al. 2014; Kong et al. 2017). Recently, one of the most promising candidates, G028.37+00.07 (C1), was removed from the list because ALMA and NOEMA observations reveal protostellar outflows driven by the core (Feng et al. 2016; Tan et al. 2016). Although a high-resolution, deep spectral imaging survey is the ultimate way to verify the starless nature, such a survey must start from a systematic sample. More candidate cores embedded within high-mass starless clumps (HMSCs) are essentially required so that some statistical understanding can be achieved. How dense clumps fragment is another key question that must be answered to understand high-mass star formation, especially in the competitive accretion scenario. Although fragments with super-Jeans masses have been revealed in some infrared dark clouds (IRDCs) with ongoing star-forming activity (e.g., Wang et al. 2011, 2014; Beuther et al. 2013, 2015; Zhang et al. 2015), whether the HMSCs can fragment into Jeans-mass cores is still a key open question. Therefore, identification and investigations of HMSCs are 
essential to understanding the formation of high-mass stars and clusters.

Recent Galactic plane surveys of dust continuum emission have revealed numerous dense structures at a wide range of evolutionary stages, including the starless phases. The APEX Telescope Large Area Survey of the Galaxy (ATLASGAL; Schuller et al. 2009), the Bolocam Galactic Plane Survey (BGPS; Aguirre et al. 2011; Dunham et al. 2011), and the Herschel Infrared Galactic Plane Survey (Hi-GAL; Molinari et al. 2010) have revealed extended dust emission at farInfrared (IR) to submillimeter, while surveys at near- to midIR, such as the Galactic Legacy Infrared Mid-Plane Survey Extraordinaire survey (GLIMPSE; Benjamin et al. 2003; Churchwell et al. 2009) and the Galactic Plane Survey using the MIPS (MIPGSGAL), reveal emissions from warm/hot dust and young stars. The combination of these surveys provides us an unprecedented opportunity to identify a large sample of candidate starless clumps.

Recently, Tackenberg et al. (2012), Traficante et al. (2015), and Svoboda et al. (2016) have identified 210, 667, and 2223 starless clump candidates in the longitude ranges $10^{\circ}<l<20^{\circ}$, $15^{\circ}<l<55^{\circ}$, and $10^{\circ}<l<65^{\circ}$, respectively. These endeavors have revealed some properties of early stages of star formation. However, these samples use criteria that do not allow them to reliably discriminate between low-mass and high-mass clumps and in some cases may have misidentified star-forming objects (see Section 3.2). Also, the covered longitude ranges are limited.

In this work, we identify a sample of HMSCs with better coverage of Galactic longitude $\left(-60^{\circ}<l<60^{\circ}\right)$ based on multiwavelength data from the GLIMPSE, MIPSGAL, HiGAL, and ATLASGAL surveys. The data used in this work are described in Section 2. The identification procedure is described in Section 3. In Section 4, we outline distance estimation and spatial distributions. Based on continuum data from 160 to $870 \mu \mathrm{m}$, some dust parameters are derived in Section 5. More in-depth discussions and a summary of the findings are given in Sections 6 and 7.

\section{Data}

This work is based on data from several Galactic plane surveys covering wavelengths from mid-IR to submillimeter. The sample of dense clumps from the ATLASGAL survey ${ }^{11}$ (Schuller et al. 2009) provides the basis for our investigation. The ATLASGAL survey mapped 420 square degrees of the Galactic plane between $-80^{\circ}<l<+60^{\circ}$, using the LABOCA camera on the $A P E X$ telescope at $870 \mu \mathrm{m}$ with a 19 !' 2 angular resolution. The astrometry of the data set and the derived source positions have been assumed to be the same as the pointing accuracy of the telescope, which is $\sim 2^{\prime \prime}-3^{\prime \prime}$ (Contreras et al. 2013). The absolute flux density uncertainty is estimated to be better than 15\% (Schuller et al. 2009). Structures larger than 2.5 have been filtered out during the reduction of the raw data because the emission from the atmosphere mimics that from extended astronomical objects. The final maps, gridded into $3^{\circ} \times 3^{\circ}$ tiles with a pixel size of $6^{\prime \prime}$, are available from the project site. ${ }^{12}$ The average noise in

\footnotetext{
11 The ATLASGAL project is a collaboration between the Max-PlanckGesellschaft, the European Southern Observatory (ESO), and the Universidad de Chile. It includes projects E-181.C-0885, E-078.F-9040(A), M-079.C-9501 (A), and M-081.C-9501(A) plus Chilean data.

12 http://atlasgal.mpifr-bonn.mpg.de/
}

the maps was determined from the $|b|<1^{\circ}$ portions of the maps to be about $70 \mathrm{mJy}_{\text {beam }^{-1}}$. On the basis of these maps, two source catalogs have been produced using the Gaussclump (Csengeri et al. 2014) and SExtractor (Contreras et al. 2013; Urquhart et al. 2014a) algorithms. The Gaussclump source catalog with 10,861 sources has been optimized for small-scale embedded structures (i.e., nearby cores and distant clumps), with background emission from molecular clouds removed (Csengeri et al. 2014). On the other hand, the 10,163 compact sources extracted using the SExtractor algorithm are representatives of larger-scale clump or cloud structures. In this work, we have used the Gaussclump source catalog of Csengeri et al. (2014) as the parent sample to identify starless clumps.

Point-source catalogs from GLIMPSE and MIPSGAL surveys have been used to identify possible young stellar object (YSO) candidates associated with ATLASGAL clumps. Using the IRAC instrument on board the Spitzer Space Telescope (Werner et al. 2004), the GLIMPSE project surveyed the inner $130^{\circ}$ of the Galactic plane at 3.6, 4.5, 5.8, and $8.0 \mu \mathrm{m}$ with $5 \sigma$ sensitivities of $0.2,0.2,0.4$, and $0.4 \mathrm{mJy}$, respectively. In addition to the images, the GLIMPSE survey performed point-source photometry, which, in combination with the Two Micron All Sky Survey point-source catalog (Skrutskie et al. 2006), provides photometric data of point sources in seven infrared bands. The MIPSGAL survey used the Multiband Infrared Photometer on Spitzer (Carey et al. 2009) to map an area comparable to GLIMPSE at longer infrared wavelengths. Version 3.0 of the MIPSGAL data includes mosaics from only the $24 \mu \mathrm{m}$ band, with a sky coverage of $|b|<1^{\circ}$ for $-68^{\circ}<l<69^{\circ}$ and $|b|<3^{\circ}$ for $-8^{\circ}<l<9^{\circ}$. The angular resolution and $5 \sigma$ sensitivity at $24 \mu \mathrm{m}$ are $6^{\prime \prime}$ and $1.7 \mathrm{mJy}$, respectively. The images and pointsource catalogs of both GLIMPSE and MIPSGAL are available at the InfraRed Science Archive (IRSA). ${ }^{13}$

Far-IR data from the Hi-GAL survey have been used to further constrain the starless clump candidates and investigate their dust properties. Hi-GAL is a key project of the Herschel Space Observatory, which mapped the entire Galactic plane with nominal $|b| \leqslant 1^{\circ}$ (following the Galactic warp). The HiGAL data were taken in fast scan mode $\left(60^{\prime \prime} \mathrm{s}^{-1}\right)$ using the PACS (70 and $160 \mu \mathrm{m})$ and SPIRE $(250,350$, and $500 \mu \mathrm{m})$ instruments in the parallel mode. The maps have been reduced with the ROMAGAL pipeline (Traficante et al. 2011), an enhanced version of the standard Herschel pipeline specifically designed for Hi-GAL. The effective angular resolutions are

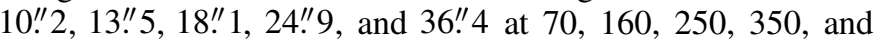
$500 \mu \mathrm{m}$, respectively.

\section{The High-mass Starless Clumps Catalog}

\subsection{Source Identification}

We have combined information from the GLIMPSE, MIPSGAL, Hi-GAL, and ATLASGAL surveys to identify starless clumps. Reliable identification requires the combination of data from all four surveys, and so only ATLASGAL clumps in the inner Galactic plane $\left(|l|<60^{\circ}\right.$ and $\left.|b|<1^{\circ}\right)$ that meet this criterion have been considered. To identify candidate HMSCs, we have applied the procedure outlined in Figure 1 to the ATLASGAL sources in the region of interest.

\footnotetext{
${ }^{13}$ http://irsa.ipac.caltech.edu
} 


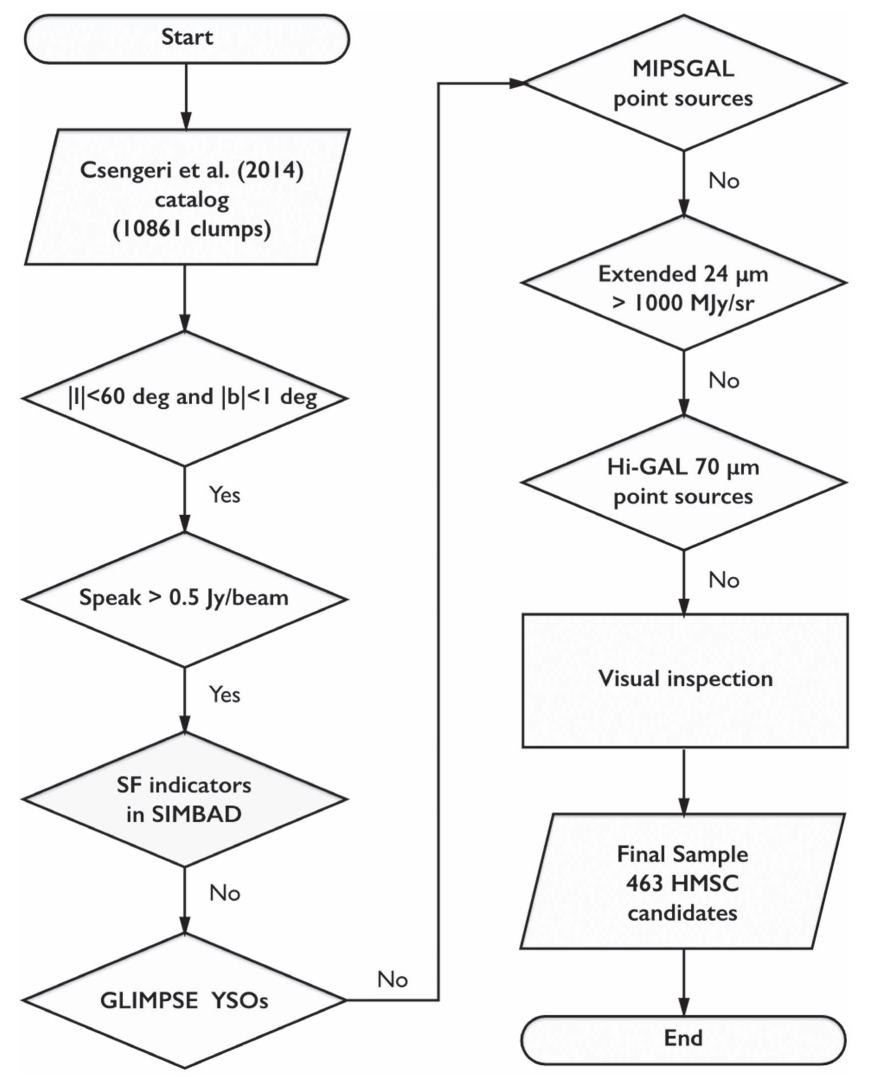

Figure 1. Flowchart describing the identification procedure of high-mass starless clumps from ATLASGAL compact sources.

As we mainly aim to find the birth sites of high-mass stars, a threshold for the peak intensity at $870 \mu \mathrm{m}$ is essential to identify high-mass clumps. Here, we followed Tackenberg et al. (2012) and select only objects with peak intensities higher than $0.5 \mathrm{Jy}_{\text {beam }}{ }^{-1}$. Assuming a dust opacity of $\kappa_{870}=1.65 \mathrm{~cm}^{2} \mathrm{~g}^{-1}$ and a dust temperature of $15 \mathrm{~K}$, this threshold corresponds to a column density of $2.2 \times 10^{22} \mathrm{~cm}^{-2}$. Although smaller than the theoretical threshold value proposed by Krumholz \& McKee (2008), which is about $1 \mathrm{~g} \mathrm{~cm}^{-2}$ or $2.15 \times 10^{23} \mathrm{~cm}^{-2}$, we consider this to be a reasonable value considering the beam dilution that will occur owing to the intermediate angular resolution of the APEX observations. The effects of distance and telescope resolution on measurements of peak column density have been studied by Vasyunina et al. (2009) under an assumed $r^{-1}$ density distribution. The peak column density at a spatial resolution of $0.01 \mathrm{pc}$ can be diluted by a factor of tens at $\sim 3 \mathrm{kpc}$ if it is observed using a single-dish telescope with an angular resolution of $11^{\prime \prime}-24^{\prime \prime}$ (Vasyunina et al. 2009). Even assuming a crude correction factor of 10, clumps with peak intensities higher than $0.5 \mathrm{Jy}^{-1}$ beam $^{-1}$ likely contain unresolved subregions with column densities higher than $2.15 \times 10^{23} \mathrm{~cm}^{-2}$.

These first two criteria reduce the number of ATLASGAL sources under consideration by more than a factor of 2 , with 5279 clumps remaining under consideration. For these clumps we have used the SIMBAD database to search for a wide range of associated star-formation-related phenomena. We queried the SIMBAD database for star-formation-associated objects within a circle defined by the major axis of the Gaussian ellipse given in Csengeri et al. (2014). If an object belonging to any of the 18 categories listed in Table 1 was found within this region, we consider the ATLASGAL source a likely star-forming
Table 1

Star-forming Indicators in SIMBAD

\begin{tabular}{ll}
\hline \hline Object Type & Object Type \\
\hline Radio source & Young stellar object \\
Centimetric radio source & Young stellar object candidate \\
H II (ionized) region & Pre-main-sequence star \\
Infrared source & Pre-main-sequence star candidate \\
Far-IR source $(\lambda \geqslant 30 \mu \mathrm{m})$ & T Tau-type star \\
Near-IR source $(\lambda<10 \mu \mathrm{m})$ & T Tau star candidate \\
Herbig-Haro object & Herbig Ae/Be star \\
Outflow & Possible Herbig Ae/Be star \\
Outflow candidate & Maser
\end{tabular}

region and exclude it from our sample. This query to SIMBAD also successfully removes clumps associated with star-forming phenomena investigated in some large surveys, such as the 6.7 GHz methanol masers (Breen et al. 2013), the red MSX sources (Lumsden et al. 2013), and H II regions (Urquhart et al. 2013).

We then further identify possible star-forming regions using data from the GLIMPSE point-source catalog and applying the color criteria given in Gutermuth et al. (2009). Any GLIMPSE source with colors that fulfill the criteria below is considered as a YSO candidate, and if any such sources lie within the region of the ATLASGAL clump, it was excluded from our starless clump sample:

$$
\begin{aligned}
{[3.6]-[4.5]>0.7 \text { and }[4.5]-[5.8]>0.7 \text { or } } \\
\left\{\begin{array}{l}
{[3.6]-[4.5]-\sigma_{1}>0.15 \text { and }} \\
{[3.6]-[5.8]-\sigma_{2}>0.35 \text { and }} \\
{[4.5]-[8.0]-\sigma_{3}>0.5 \text { and }} \\
{[3.6]-[5.8]+\sigma_{2} \leqslant \frac{0.14}{0.04}} \\
\times\left(\left([4.5]-[8.0]-\sigma_{3}\right)-0.5\right)+0.5
\end{array}\right.
\end{aligned}
$$

Here $\sigma_{1}=\sigma([3.6]-[4.5]), \sigma_{2}=\sigma([3.6]-[5.8])$, and $\sigma_{3}=\sigma$ $([4.5]-[8.0])$ are combined errors, added in quadrature.

In a recent study, Gallaway et al. (2013) show that $17 \%$ of methanol masers from the Methanol Multi-Beam (MMB) survey are not associated with emission seen in GLIMPSE, indicating that some very young high-mass stars may be too cold to be detectable in IRAC bands but show weak $24 \mu \mathrm{m}$ emission. Thus, any clump that is associated with a $24 \mu \mathrm{m}$ point source probably hosts starforming activity and should be omitted. By querying the VizieR Service, ${ }^{14}$ we seized and excluded clumps that are possibly associated with $24 \mu \mathrm{m}$ point sources provided in Gutermuth \& Heyer (2015). Furthermore, some very faint point sources at $24 \mu \mathrm{m}$ could have been missed from the catalog of Gutermuth \& Heyer (2015), due to bright extended emission contamination. In order to obtain a reliable sample of HMSCs, we also have ignored clumps associated with $24 \mu \mathrm{m}$ extended structures brighter than $10^{3} \mathrm{MJy} \mathrm{sr}^{-1}$. This threshold corresponds to about $2.0 \mathrm{mag}$ within an aperture of $6 . " 35$, close to the $90 \%$ differential completeness limit ( $\sim 1.98 \mathrm{mag}$ ) of $24 \mu \mathrm{m}$ point sources in bright and structured regions (Gutermuth \& Heyer 2015).

A total of 1215 ATLASGAL compact sources remain after applying these exclusion criteria. These clumps were then subjected to a final visual inspection to identify (and exclude) any that have very faint $24 \mu \mathrm{m}$ point emission missed from the

\footnotetext{
${ }^{14}$ http://vizier.u-strasbg.fr
} 
Table 2

Basic Parameters of HMSC Candidates

\begin{tabular}{|c|c|c|c|c|c|c|c|c|c|c|}
\hline Designation & R.A. & Decl. & $\begin{array}{c}\Theta_{\mathrm{maj}} \\
(\operatorname{arcsec})\end{array}$ & $\begin{array}{c}\Theta_{\min } \\
(\operatorname{arcsec})\end{array}$ & $\begin{array}{c}\mathrm{PA}^{\mathrm{a}} \\
(\mathrm{deg})\end{array}$ & $\begin{array}{l}\text { FWHM } \\
(\operatorname{arcsec})\end{array}$ & $\begin{array}{c}V_{\mathrm{lsr}} \\
\left(\mathrm{km} \mathrm{s}^{-1}\right)\end{array}$ & $\begin{array}{c}\text { Distance } \\
(\mathrm{kpc})\end{array}$ & Reference $^{b}$ & $70 \mu \mathrm{m} \mathrm{Dark}{ }^{\mathrm{c}}$ \\
\hline G000.3404+0.0562 & $17^{\mathrm{h}} 46^{\mathrm{m}} 12^{\mathrm{s}} \cdot 62$ & $-28^{\mathrm{d}} 36^{\mathrm{m}} 58^{\mathrm{s}} \cdot 3$ & 45 & 30 & 163 & 37 & -11.80 & $4.19(0.22)$ & $\mathrm{JCO}$ & $\mathrm{Y}$ \\
\hline G003.2278+0.4924 & $17^{\mathrm{h}} 51^{\mathrm{m}} 13^{\mathrm{s}} \cdot 61$ & $-25^{\mathrm{d}} 55^{\mathrm{m}} 02^{\mathrm{s}} .3$ & 55 & 30 & 90 & 41 & 16.10 & $2.93(0.15)$ & SMT & $\mathrm{N}$ \\
\hline G006.2130-0.5937 & $18^{\mathrm{h}} 01^{\mathrm{m}} 59^{\mathrm{s}} .02$ & $-23^{\mathrm{d}} 52^{\mathrm{m}} 55^{\mathrm{s}} .2$ & 39 & 20 & 128 & 27 & 18.40 & $2.95(0.15)$ & w12 & $\mathrm{N}$ \\
\hline G008.1102+0.2591 & $18^{\mathrm{h}} 02^{\mathrm{m}} 49^{\mathrm{s}} .73$ & $-21^{\mathrm{d}} 48^{\mathrm{m}} 40^{\mathrm{s}} \cdot 0$ & 25 & 23 & 8 & 24 & 18.90 & $2.98(0.22)$ & $\mathrm{c} 14$ & $\mathrm{~N}$ \\
\hline G008.5441-0.3421 & $18^{\mathrm{h}} 06^{\mathrm{m}} 00^{\mathrm{s}} \cdot 12$ & $-21^{\mathrm{d}} 43^{\mathrm{m}} 41^{\mathrm{s}} \cdot 2$ & 34 & 26 & 151 & 30 & 37.10 & $4.45(0.23)$ & w12 & $\mathrm{N}$ \\
\hline G008.7264-0.3959 & $18^{\mathrm{h}} 06^{\mathrm{m}} 35^{\mathrm{s}} .23$ & $-21^{\mathrm{d}} 35^{\mathrm{m}} 42^{\mathrm{s}} .7$ & 28 & 21 & 137 & 24 & 39.10 & $4.47(0.24)$ & $\mathrm{c} 14$ & $\mathrm{~N}$ \\
\hline G010.0676-0.4076 & $18^{\mathrm{h}} 09^{\mathrm{m}} 25^{\mathrm{s}} .37$ & $-20^{\mathrm{d}} 25^{\mathrm{m}} 43^{\mathrm{s}} .0$ & 36 & 24 & 101 & 29 & 11.40 & $3.11(0.21)$ & s13 & $\mathrm{N}$ \\
\hline G010.1065-0.4168 & $18^{\mathrm{h}} 09^{\mathrm{m}} 32^{\mathrm{s}} \cdot 26$ & $-20^{\mathrm{d}} 23^{\mathrm{m}} 56^{\mathrm{s}} \cdot 4$ & 41 & 27 & 27 & 33 & 11.50 & $3.11(0.21)$ & w12 & $\mathrm{N}$ \\
\hline G010.1839-0.4050 & $18^{\mathrm{h}} 09^{\mathrm{m}} 39^{\mathrm{s}} \cdot 19$ & $-20^{\mathrm{d}} 19^{\mathrm{m}} 32^{\mathrm{s}} .2$ & 34 & 20 & 38 & 26 & 15.30 & $3.12(0.21)$ & $\mathrm{c} 14$ & $\mathrm{~N}$ \\
\hline G012.8572-0.2088 & $18^{\mathrm{h}} 14^{\mathrm{m}} 21^{\mathrm{s}} \cdot 94$ & $-17^{\mathrm{d}} 53^{\mathrm{m}} 13^{\mathrm{s}} \cdot 9$ & 31 & 25 & 177 & 28 & 32.70 & $3.00(0.31)$ & d13 & $\mathrm{Y}$ \\
\hline G018.8441-0.3758 & $18^{\mathrm{h}} 26^{\mathrm{m}} 41^{\mathrm{s}} .30$ & $-12^{\mathrm{d}} 41^{\mathrm{m}} 11^{\mathrm{s}} .8$ & 60 & 34 & 14 & 45 & 61.00 & $3.60(0.26)$ & s13 & $\mathrm{Y}$ \\
\hline G018.9295-0.0289 & $18^{\mathrm{h}} 25^{\mathrm{m}} 35^{\mathrm{s}} .64$ & $-12^{\mathrm{d}} 26^{\mathrm{m}} 57^{\mathrm{s}} .1$ & 48 & 37 & 45 & 42 & 43.60 & $3.33(0.18)$ & p12 & $\mathrm{Y}$ \\
\hline G320.2715+0.2920 & $15^{\mathrm{h}} 07^{\mathrm{m}} 56^{\mathrm{s}} .06$ & $-57^{\mathrm{d}} 54^{\mathrm{m}} 33^{\mathrm{s}} .5$ & 33 & 21 & 44 & 26 & -32.10 & $10.80(0.49)$ & j08 & $\mathrm{Y}$ \\
\hline G320.3385-0.1534 & $15^{\mathrm{h}} 10^{\mathrm{m}} 04^{\mathrm{s}} .01$ & $-58^{\mathrm{d}} 15^{\mathrm{m}} 38^{\mathrm{s}} .5$ & 24 & 20 & 121 & 22 & -9.00 & $12.24(0.51)$ & u 14 & $\mathrm{Y}$ \\
\hline G326.4923+0.8820 & $15^{\mathrm{h}} 42^{\mathrm{m}} 37^{\mathrm{s}} \cdot 99$ & $-53^{\mathrm{d}} 58^{\mathrm{m}} 02^{\mathrm{s}} \cdot 6$ & 32 & 24 & 108 & 28 & -39.30 & $2.46(0.45)$ & j08 & $\mathrm{Y}$ \\
\hline G333.0151-0.4964 & $16^{\mathrm{h}} 20^{\mathrm{m}} 48^{\mathrm{s}} .00$ & $-50^{\mathrm{d}} 43^{\mathrm{m}} 01^{\mathrm{s}} .2$ & 47 & 26 & 101 & 35 & -56.40 & $3.54(0.42)$ & $\mathrm{u} 07$ & $\mathrm{~N}$ \\
\hline G333.1639-0.4413 & $16^{\mathrm{h}} 21^{\mathrm{m}} 13^{\mathrm{s}} \cdot 22$ & $-50^{\mathrm{d}} 34^{\mathrm{m}} 22^{\mathrm{s}} \cdot 4$ & 32 & 20 & 17 & 25 & -52.60 & $3.36(0.43)$ & $\mathrm{c} 14$ & $\mathrm{~N}$ \\
\hline G336.4689-0.2023 & $16^{\mathrm{h}} 34^{\mathrm{m}} 13^{\mathrm{s}} .27$ & $-48^{\mathrm{d}} 01^{\mathrm{m}} 30^{\mathrm{s}} .0$ & 47 & 22 & 62 & 32 & -24.30 & $13.44(0.55)$ & $\mathrm{c} 14$ & $\mathrm{~N}$ \\
\hline G336.7428+0.1078 & $16^{\mathrm{h}} 33^{\mathrm{m}} 58^{\mathrm{s}} \cdot 22$ & $-47^{\mathrm{d}} 36^{\mathrm{m}} 48^{\mathrm{s}} \cdot 2$ & 36 & 28 & 69 & 32 & -76.30 & $10.66(0.41)$ & $\mathrm{m} 90$ & $\mathrm{~N}$ \\
\hline $\mathrm{G} 359.9214+0.0276$ & $17^{\mathrm{h}} 45^{\mathrm{m}} 19^{\mathrm{s}} .51$ & $-28^{\mathrm{d}} 59^{\mathrm{m}} 20^{\mathrm{s}} 0$ & 40 & 40 & 59 & 40 & 59.30 & $10.90(0.24)$ & $\mathrm{T} 13 \mathrm{CO}$ & $\mathrm{N}$ \\
\hline
\end{tabular}

Notes.

a Position angle corrected in the FK5 system with respect to the north direction.

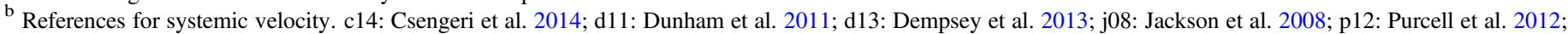

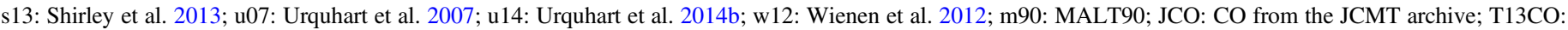

${ }^{13} \mathrm{CO}$ from the ThrUMMS survey; SMT: single-point observations using SMT.

c "Y" indicates that the clump is associated with the extinction feature at $70 \mu \mathrm{m}$.

(This table is available in its entirety in machine-readable form.)

Gutermuth \& Heyer (2015) catalog, any that are saturated at $24 \mu \mathrm{m}$, and any that are associated with $70 \mu \mathrm{m}$ point-like sources (Molinari et al. 2016b).

In the procedures of removing star-forming clumps, some star-forming activities could be in the foreground, leading to underestimating the number of starless clumps. However, the main goal of this work is to identify a more reliable sample of HMSCs, not a complete catalog. The resulting sample contains 463 HMSC candidates, which are listed in Table 2. We find that most of the HMSC candidates are associated with IRDCs; about $49 \%(229 / 463)$ are even dark at $70 \mu \mathrm{m}$ (see Figure 2 and column (11) of Table 2). Further inspection using far-IR continuum data supports the dense and cold nature of these sources (see Sections 5 and 6).

\subsection{Comparison with Reported Starless Clump Catalogs}

There are three previous papers that have searched for starless clumps. Tackenberg et al. (2012) reported a sample of 210 starless clumps in the $10^{\circ}<l<20^{\circ}$ range. Star-forming clumps were ruled out via identifying YSOs from the GLIMPSE catalog based on IR colors and visual analysis of $24 \mu \mathrm{m}$ images (Tackenberg et al. 2012). Loose criteria for filtering out star-forming clumps led to a greater than $50 \%$ misidentification in the Tackenberg et al. (2012) catalog (Svoboda et al. 2016). Among the 210 starless clumps of Tackenberg et al. (2012), only 107 have counterparts in the ATLASGAL GaussClump Catalog (Csengeri et al. 2014), and more than $70 \%(75 / 107)$ are possible star-forming clumps if diagnosed using the criteria applied in this work.

In the $15^{\circ}<l<55^{\circ}$ and $|b|<1^{\circ}$ area, Traficante et al. (2015) identified 667 starless clumps in IRDCs based on
Hi-GAL data. The Hyper algorithm was used for clump extraction, and counterparts at $70 \mu \mathrm{m}$ were used for protostellar identification. Spatial cross-matching shows that 175 starless clumps from Traficante et al. (2015) have counterparts in the ATLASGAL catalog, and only $20(\sim 11 \%)$ are associated with HMSC candidates identified in this work. The remaining $89 \%$ $(155 / 175)$ possibly can only form low-mass stars or already host star-forming activity. Traficante et al. (2015) suggested that about $26 \%$ of their starless clumps have the potential to form high-mass stars. This suggests that about $50 \%$ of starless clumps reported in Traficante et al. (2015) may already have started forming stars.

Another catalog of starless clumps has been compiled by Svoboda et al. (2016) based on Bolocam survey data. Starforming indicators including mid- to far-IR YSOs, masers, and ultracompact $\mathrm{H}$ II regions were considered to rule out clumps with current star formation. Among the 2223 starless clumps in $10^{\circ}<l<65^{\circ}$ from Svoboda et al. (2016), 179 have counterparts in the ATLASGAL catalog, and 35 (20\%) are associated with HMSC candidates identified in this work. As noted in Svoboda et al. (2016), about $10 \%$ of their starless clumps have the potential to form high-mass stars.

In brief, we may have singled out the by far largest sample of relatively reliable HMSC candidates distributed throughout the whole inner Galactic plane.

\section{Distances and Spatial Distribution}

\subsection{Distance Estimation}

The distance to a source is a fundamental parameter that is essential to determine its mass and luminosity. The distance to 

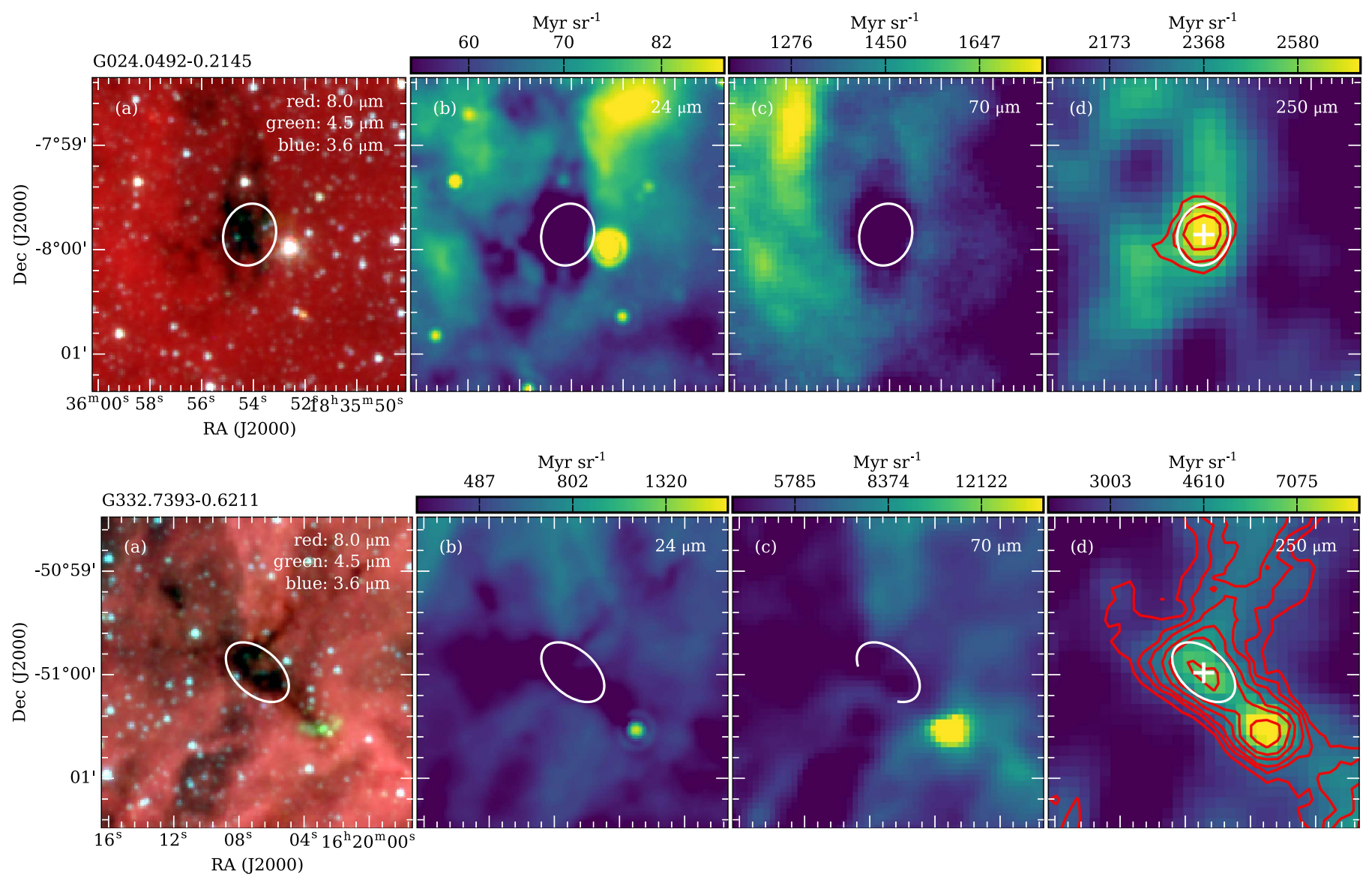

Figure 2. Morphology of two example HMSCs in different wavelength bands. (a) Three-color images with emission at 8.0, 4.5, and 3.6 $\mu \mathrm{m}$ rendered in red, green, and blue, respectively. (b), (c), (d) show dust continuum emission at 24, 70, and $250 \mu \mathrm{m}$, respectively. In panel (d), emission at $870 \mu \mathrm{m}$ is presented in red contours with levels of $0.3,0.4,0.5,0.7,0.9,1.3,1.8,2.5,4,7 \mathrm{Jy} \mathrm{beam}^{-1}$. The white plus sign gives the peak position of $870 \mu \mathrm{m}$, and the white ellipse delineates the source size based on the major and minor half-intensity axes provided in the ATLASGAL catalog.

(The complete figure set (463 images) is available.)

the HMSC candidates is not known for most sources, and to address this, we have collected the systemic velocities from the literature for 294 clumps. For a further 20, 63, and 58 sources, velocity measurements have been obtained based on data collected from the MALT90 survey (Jackson et al. 2013), the ThrUMMS survey (Barnes et al. 2015), and the JCMT archive. Velocities for the remaining 28 sources were determined via single-point observations of ${ }^{13} \mathrm{CO}(2-1)$ or $\mathrm{C}^{18} \mathrm{O}(2-1)$ using the Submillimeter Telescope (SMT) of the Arizona Radio Observatory. For all of the HMSCs, distance estimates were obtained using a parallax-based distance estimator. ${ }^{15}$ Leveraging results of trigonometric parallaxes from the BeSSeL (Bar and Spiral Structure Legacy Survey) and VERA (Japanese VLBI Exploration of Radio Astrometry) projects, the distance estimator can reasonably resolve kinematic distance ambiguities based on the Bayesian approach (Reid et al. 2016). The distances calculated using the FORTRAN version of the estimator are given in column (9) of Table 2. The $463 \mathrm{HMSCs}$ have distances ranging from 0.3 to $18.3 \mathrm{kpc}$ with a mean of 7.1 $\mathrm{kpc}$ and a median of $6.0 \mathrm{kpc}$.

The equivalent radius $\left(r_{\text {eq }}\right)$ of the ATLASGAL clumps was estimated by multiplying the distance by the deconvolved equivalent angular radius $\theta_{\mathrm{eq}}=\sqrt{\Theta_{\text {maj }} \Theta_{\text {min }}-\theta_{\mathrm{HPBW}}^{2}}$, where

\footnotetext{
${ }^{15}$ http://bessel.vlbi-astrometry.org/bayesian
}

$\theta_{\text {HPBW }}=19$ !' 2 is the ATLASGAL beam. The major and minor half-intensity axes $\left(\Theta_{\text {maj }}\right.$ and $\left.\Theta_{\text {maj }}\right)$ were obtained from Csengeri et al. (2014). The resultant equivalent radii are given in column (2) of Table 3 . The HMSC candidates have a median equivalent radius of $0.65 \mathrm{pc}$, consistent with that of clumps identified in some IRDCs ( $\sim 0.6$ pc; Traficante et al. 2015$)$ and that of BGPS clumps ( 0.64 pc; Svoboda et al. 2016).

\subsection{Spatial Distribution}

The distributions of the identified HMSC candidates in the inner Galactic plane and as a function of Galactic longitude are shown in Figures 3 and 4, respectively. The distribution of HMSCs in Galactic longitude is very similar to that of the full sample of ATLASGAL clumps. A prominent overdensity can be observed toward the Galactic center region. In addition, there are several well-known star-forming complexes (i.e., W51, W43, G23, M17, W31/W33, NGC 6334/6357, G337, G333, G327, and G305) standing out with significant peaks. Many of the HMSCs are associated with large-scale filaments identified by Wang et al. (2016). In the longitude range $7.5^{\circ}<l<60^{\circ}$ covered by both Wang et al. (2016) and this study, there are 145 HMSCs, 39 of which are located on large-scale filaments. This implies that about $27 \%(39 / 145)$ of future high-mass star formation would take place in large-scale $(>10 \mathrm{pc})$ filamentary structures in the Galactic plane. 
Table 3

Physical Parameters of HMSC Candidates

\begin{tabular}{|c|c|c|c|c|c|c|c|c|}
\hline Designation & $\begin{array}{c}r_{\mathrm{eq}} \\
(\mathrm{pc})\end{array}$ & $\begin{array}{l}T_{\text {dust }} \\
(\mathrm{K})\end{array}$ & $\begin{array}{c}N_{\mathrm{H}_{2}} \\
\left(10^{22} \mathrm{~cm}^{-2}\right)\end{array}$ & $\begin{array}{c}n_{\mathrm{H}_{2}} \\
\left(10^{4} \mathrm{~cm}^{-3}\right)\end{array}$ & $\underset{\left(\mathrm{g} \mathrm{cm}^{-2}\right)}{\Sigma_{\text {mass }}}$ & $\begin{array}{c}M_{\mathrm{cl}} \\
\left(M_{\odot}\right)\end{array}$ & $\begin{array}{c}L_{\mathrm{cl}} \\
\left(L_{\odot}\right)\end{array}$ & $\begin{array}{c}L_{\mathrm{cl}} / M_{\mathrm{cl}} \\
\left(L_{\odot} / M_{\odot}\right)\end{array}$ \\
\hline G000.3404+0.0562 & 0.64 & 17.08 & 18.44 & 3.26 & 0.40 & $2.44 \mathrm{e}+03$ & $4.83 e+03$ & 1.98 \\
\hline G003.2278+0.4924 & 0.51 & 14.37 & 3.14 & 0.61 & 0.06 & $2.34 \mathrm{e}+02$ & $1.88 \mathrm{e}+02$ & 0.80 \\
\hline G006.2130-0.5937 & 0.29 & 15.75 & 3.49 & 2.36 & 0.13 & $1.67 \mathrm{e}+02$ & $2.44 \mathrm{e}+02$ & 1.46 \\
\hline G008.1102+0.2591 & 0.21 & 18.41 & 2.72 & 5.13 & 0.21 & $1.33 \mathrm{e}+02$ & $4.76 \mathrm{e}+02$ & 3.58 \\
\hline G008.5441-0.3421 & 0.49 & 12.32 & 4.23 & 1.25 & 0.12 & $4.27 \mathrm{e}+02$ & $1.41 \mathrm{e}+02$ & 0.33 \\
\hline G008.7264-0.3959 & 0.32 & 12.52 & 6.88 & 7.86 & 0.49 & $7.54 \mathrm{e}+02$ & $2.48 \mathrm{e}+02$ & 0.33 \\
\hline G010.0676-0.4076 & 0.34 & 18.78 & 2.04 & 1.13 & 0.07 & $1.24 \mathrm{e}+02$ & $5.48 \mathrm{e}+02$ & 4.43 \\
\hline G010.1065-0.4168 & 0.41 & 18.38 & 2.71 & 0.82 & 0.06 & $1.64 \mathrm{e}+02$ & $6.05 \mathrm{e}+02$ & 3.69 \\
\hline G010.1839-0.4050 & 0.27 & 22.43 & 1.77 & 1.55 & 0.08 & $8.55 \mathrm{e}+01$ & $1.20 \mathrm{e}+03$ & 13.98 \\
\hline G012.8572-0.2088 & 0.29 & 17.35 & 5.31 & 3.65 & 0.21 & $2.67 \mathrm{e}+02$ & $6.60 \mathrm{e}+02$ & 2.47 \\
\hline G018.8441-0.3758 & 0.71 & 14.30 & 4.38 & 0.58 & 0.08 & $6.09 \mathrm{e}+02$ & $5.12 \mathrm{e}+02$ & 0.84 \\
\hline G018.9295-0.0289 & 0.61 & 18.18 & 3.22 & 0.52 & 0.06 & $3.36 \mathrm{e}+02$ & $1.40 \mathrm{e}+03$ & 4.16 \\
\hline G320.2715+0.2920 & 0.94 & 12.15 & 2.87 & 0.74 & 0.13 & $1.81 \mathrm{e}+03$ & $5.19 \mathrm{e}+02$ & 0.29 \\
\hline G320.3385-0.1534 & 0.63 & 16.00 & 4.16 & 4.82 & 0.58 & $3.43 \mathrm{e}+03$ & $5.52 \mathrm{e}+03$ & 1.61 \\
\hline G326.4923+0.8820 & 0.24 & 13.88 & 8.22 & 7.26 & 0.33 & $2.85 \mathrm{e}+02$ & $1.76 \mathrm{e}+02$ & 0.62 \\
\hline G333.0151-0.4964 & 0.50 & 17.60 & 5.45 & 1.60 & 0.16 & $5.86 \mathrm{e}+02$ & $1.37 \mathrm{e}+03$ & 2.34 \\
\hline G333.1639-0.4413 & 0.27 & 18.91 & 6.84 & 7.31 & 0.38 & $4.10 \mathrm{e}+02$ & $1.69 \mathrm{e}+03$ & 4.13 \\
\hline G336.4689-0.2023 & 1.68 & 14.19 & 2.25 & 0.21 & 0.07 & $2.85 \mathrm{e}+03$ & $2.20 \mathrm{e}+03$ & 0.77 \\
\hline G336.7428+0.1078 & 1.31 & 22.16 & 1.54 & 0.16 & 0.04 & $1.02 \mathrm{e}+03$ & $1.42 \mathrm{e}+04$ & 13.86 \\
\hline G359.9214+0.0276 & 1.85 & 18.56 & 6.24 & 0.32 & 0.11 & $5.88 \mathrm{e}+03$ & $2.09 \mathrm{e}+04$ & 3.56 \\
\hline
\end{tabular}

(This table is available in its entirety in machine-readable form.)

To determine the locations of these HMSCs in our Galaxy, we have plotted the sources on a top-down schematic of the Milky Way (see Figure 5). Most targets are located in spiral arms in the inner Galaxy with galactocentric distances $R_{\text {gal }}<8.34 \mathrm{kpc}$. Most sources in the general direction of W51 $\left(l \sim 49^{\circ}\right)$ are located in the Carina-Sagittarius arm, with an average distance of about $6 \mathrm{kpc}$, consistent with that of the W51 complex (Sato et al. 2010). Sources toward W43 $\left(l \sim 31^{\circ}\right)$ and G23 $\left(l \sim 23^{\circ}\right)$ mostly reside in the Scutum-Centaurus arm, and some in the near $3 \mathrm{kpc}$ arm. The overdensities at $l=10^{\circ}-17^{\circ}$ are associated with the W31 and W33 complexes and the well-known starforming region M17. The sources in this Galactic longitude range are mostly located in the Scutum-Centaurus arm, with some in the Perseus and Norma arms with distances larger than $10 \mathrm{kpc}$. The peak at $l=352^{\circ}$ originates from two groups of sources, with the near group associated with the relatively nearby complex of NGC 6334/6357 (Russeil et al. 2010) located in the Carina-Sagittarius arm and the far group located in the Galactic bar. The peaks toward G327, G333, and G337 are mostly located in the Scutum-Centaurus arm, and some in the Perseus, Norma, and near $3 \mathrm{kpc}$ arms. The sources in the Crux-Scutum arm at this direction have an average distance of about $3.5 \mathrm{kpc}$, consistent with that of the G333.2-0.4 giant molecular cloud (Simpson et al. 2012). Sources at $l=305^{\circ}$ are mostly located at the tangent point of the Crux-Scutum arm.

\section{Dust Properties}

For all 463 HMSC candidates, there are image data at six farIR to submillimeter bands covering wavelengths from 70 to $870 \mu \mathrm{m}$ collected by the Hi-GAL and ATLASGAL surveys, enabling us to obtain some physical parameters. As these sources effectively do not emit at $70 \mu \mathrm{m}$, we only take emission at 160 , $250,350,500$, and $870 \mu \mathrm{m}$ into account to obtain physical parameters.

\subsection{Convolution to a Common Resolution and Foreground/ Background Filtering}

In order to be able to estimate physical properties from multiwavelength observations with different angular resolutions, we first convolved the images to a common angular resolution of 36!" 4 , which is essentially the poorest resolution of any of the wavelengths. The convolution package of Astropy (Astropy Collaboration et al. 2013) was used with a Gaussian kernel of $\sqrt{366^{\prime \prime} 4-\theta_{\lambda}^{2}}$, where $\theta_{\lambda}$ is the HPBW beam size for a given Hi-GAL or ATLASGAL band. Then the convolved data from the different bands were regridded to be aligned pixel by pixel with a common pixel size of 11 " 5 .

As our targets are in the Galactic plane, foreground/background removal is essential to reduce the uncertainties in some of the derived parameters. For the ATLASGAL images, any uniform astronomical signal on spatial scales larger than 2.5 has been filtered out together with atmospheric emission during the data reduction (Schuller et al. 2009). The filtering of Hi-GAL images was performed using the CUPID-findback algorithm of the Starlink suite. ${ }^{16}$ The algorithm constructs the background iteratively from the original image. At first, a filtered form of the input data is produced by replacing every input pixel by the minimum of the input values within a rectangular box centered on the pixel. This filtered data are then filtered again, using a filter that replaces every pixel value by the maximum value in a box centered on the pixel. Then each pixel in these filtered data is replaced by the mean value in a box centered on the pixel. The same box size is used for the first three steps. The final background estimate is obtained via some corrections and iterations through comparison with the initial input data. For further details on the algorithm please see the online document for findback. ${ }^{17}$ As a key parameter, the filtering box was chosen to be

\footnotetext{
16 http://starlink.eao.hawaii.edu/starlink/WelcomePage

17 http://starlink.eao.hawaii.edu/starlink/findback.html
} 

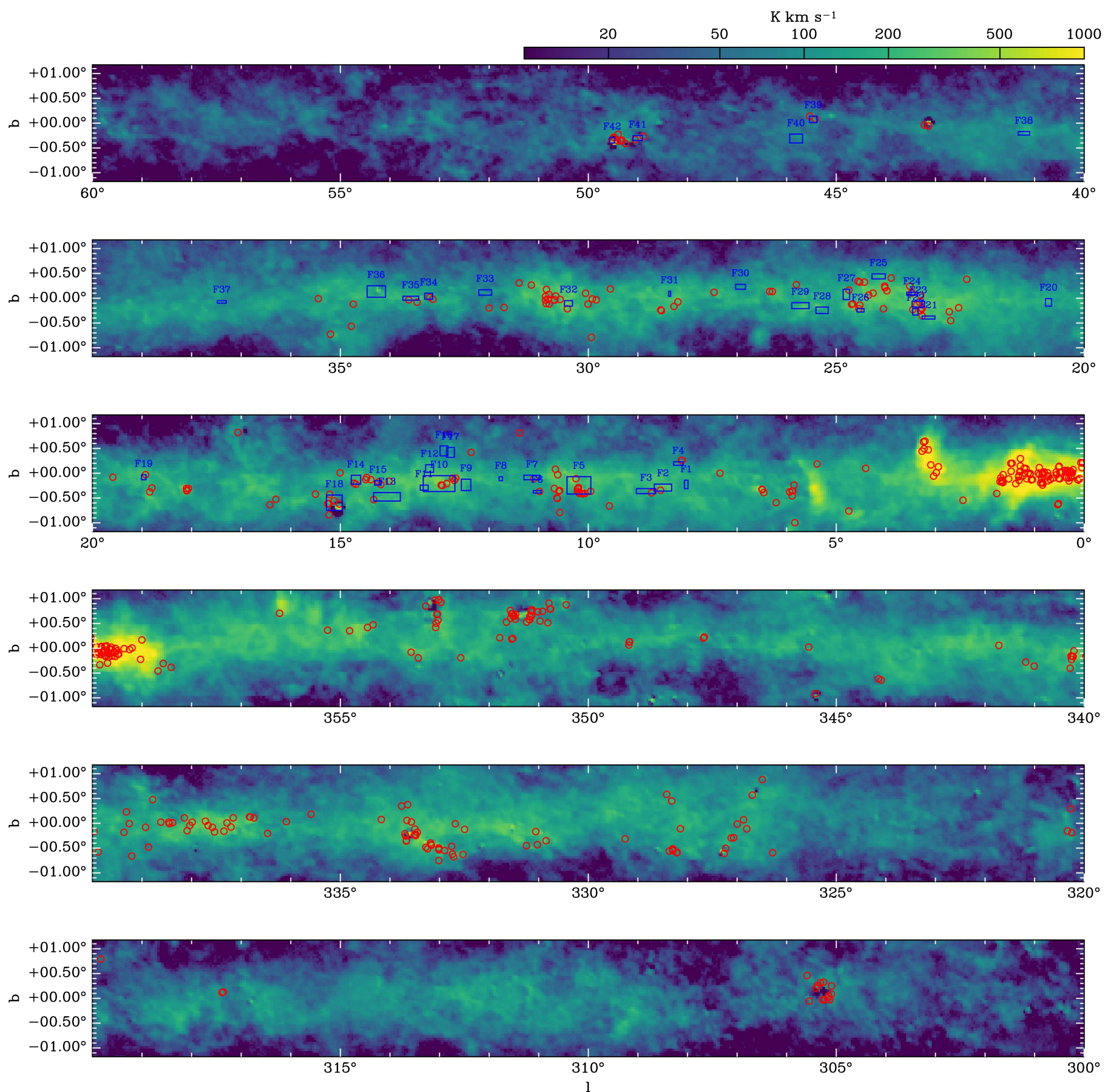

Figure 3. Distribution of HMSC candidates in the inner Galactic plane. The background shows CO $J=1-0$ emission from Planck Collaboration et al. (2014). The open boxes delineate the large-scale dense filaments identified by Wang et al. (2016).

$2 ! 5$ for consistency with the ATLASGAL data. One drawback of the findback algorithm is that the background can be overestimated when processing high signal-to-noise data, especially when there are strong emission features significantly smaller than the box size. To mitigate issues with overestimation of the background level, we set the parameter NEWALG to TRUE and iteratively ran findback. The background image resulting from the previous run was used as the input data for the next iteration. After careful examination of a number of test sources, we found that the resulting background images became stable after five iterations. The background images after five iterative runs of findback were subtracted from the post-convolution data for all Hi-GAL bands to remove large-scale structures.

\subsection{Spectral Energy Distribution Fitting}

We have used the smoothed and background-removed far-IR to submillimeter image data to obtain intensity as a function of wavelength for each pixel and applied a modified blackbody model to these data:

$$
I_{\nu}=B_{\nu}(T)\left(1-e^{-\tau_{\nu}}\right)
$$




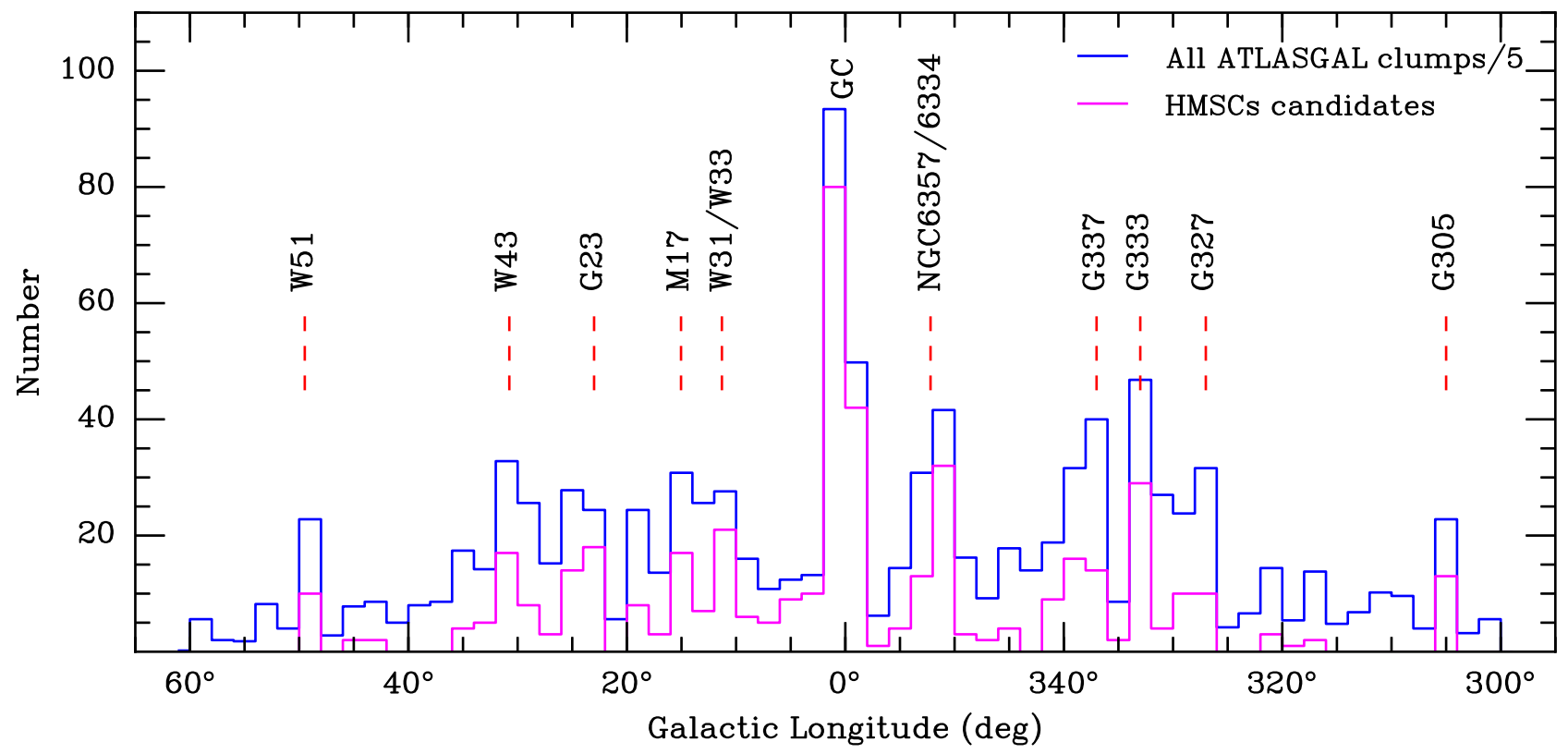

Figure 4. Histograms of Galactic longitudes of the HMSC candidates and all ATLASGAL clumps. Note that the number of sources in each bin for the full ATLASGAL catalog has been scaled down by a factor of five.

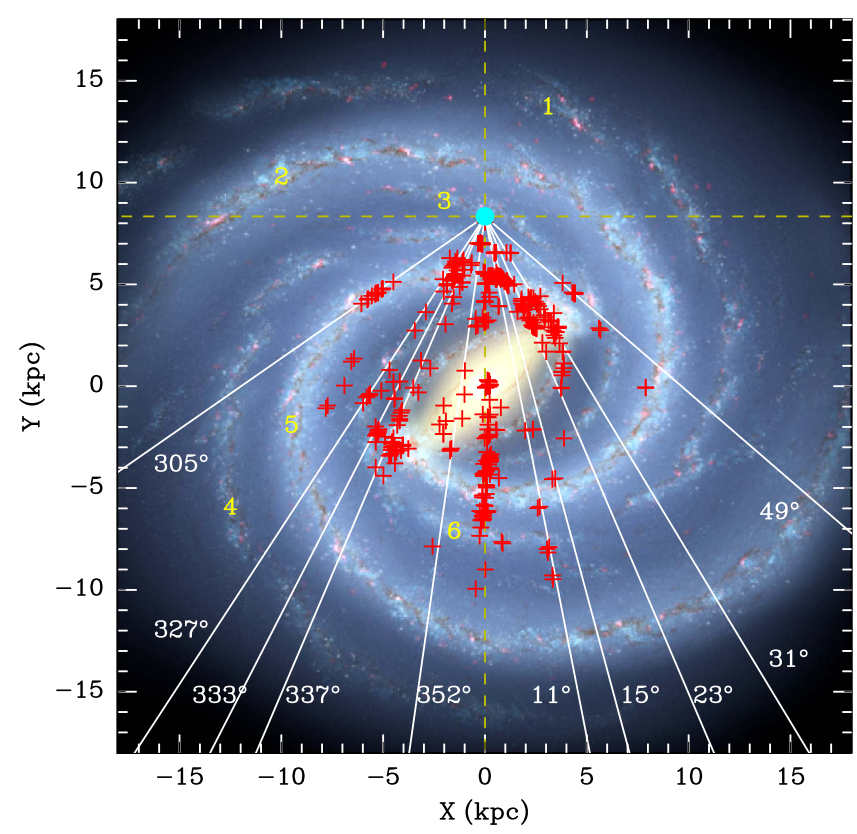

Figure 5. Spatial distribution of the identified HMSCs projected onto a topdown schematic of the Milky Way (artist's concept, R. Hurt: NASA/JPLCaltech/SSC). The spiral arms are indicated using numbers from 1 to 6 , referring to the Outer, Perseus, Local, Carina-Sagittarius, Scutum-Centaurus, and Norma arms. (Codes and FITS files for making this plot are available from https://github.com/yuanjinghua/Top_Down_Miky_Way.)

where the Planck function $B_{\nu}(T)$ is modified by optical depth

$$
\tau_{\nu}=\mu_{\mathrm{H}_{2}} m_{\mathrm{H}} \kappa_{\nu} N_{\mathrm{H}_{2}} / R_{\mathrm{gd}}
$$

Here $\mu_{\mathrm{H}_{2}}=2.8$ is the mean molecular weight adopted from Kauffmann et al. (2008), $m_{\mathrm{H}}$ is the mass of a hydrogen atom, $N_{\mathrm{H}_{2}}$ is the $\mathrm{H}_{2}$ column density, and $R_{\mathrm{gd}}=100$ is the gas-to-dust ratio. The dust opacity $\kappa_{\nu}$ can be expressed as a power law in
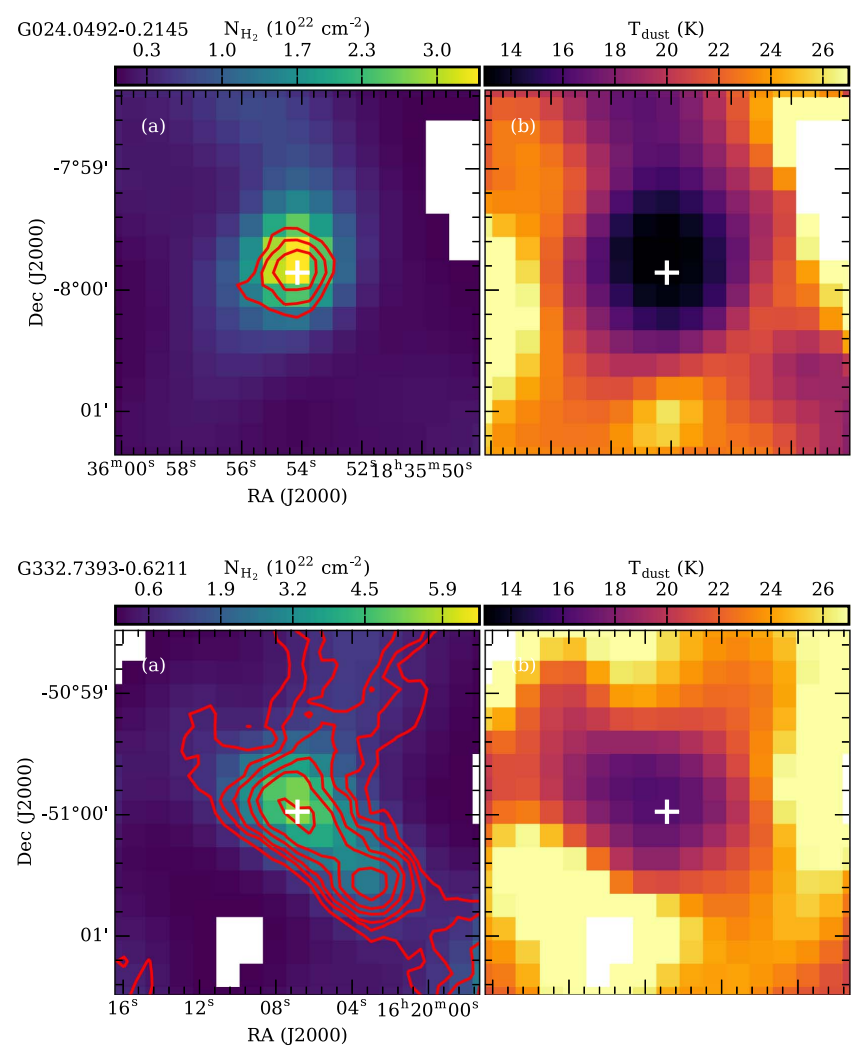

Figure 6. Column density (a) and dust temperature maps (b) of two exemplary HMSC candidates. The contours are the same as the ones in Figure 2(d).

(The complete figure set (463 images) is available.)

frequency,

$$
\kappa_{\nu}=3.33\left(\frac{\nu}{600 \mathrm{GHz}}\right)^{\beta} \mathrm{cm}^{2} \mathrm{~g}^{-1}
$$


Table 4

Statistics of Some Parameters for Clumps at Different Stages

\begin{tabular}{|c|c|c|c|c|c|c|c|c|c|}
\hline Statistic & $\begin{array}{l}\text { Distance } \\
(\mathrm{kpc})\end{array}$ & $\begin{array}{l}r_{\mathrm{eq}} \\
(\mathrm{pc})\end{array}$ & $\begin{array}{l}T_{\text {dust }} \\
(\mathrm{K})\end{array}$ & $\begin{array}{c}N_{\mathrm{H}_{2}} \\
\left(10^{22} \mathrm{~cm}^{-2}\right)\end{array}$ & $\begin{array}{c}n_{\mathrm{H}_{2}} \\
\left(10^{4} \mathrm{~cm}^{-3}\right)\end{array}$ & $\begin{array}{c}\sum_{\text {mass }} \\
\left(\mathrm{g} \mathrm{cm}^{-2}\right)\end{array}$ & $\begin{array}{c}M_{\mathrm{cl}} \\
\left(M_{\odot}\right)\end{array}$ & $\begin{array}{c}L_{\mathrm{cl}} \\
\left(L_{\odot}\right)\end{array}$ & $\begin{array}{c}L_{\mathrm{cl}} / M_{\mathrm{cl}} \\
\left(L_{\odot} / M_{\odot}\right)\end{array}$ \\
\hline \multicolumn{10}{|c|}{ Starless Clumps } \\
\hline Max & 18.3 & 3.57 & 24.96 & 64.07 & 236.42 & 2.14 & $6.1 \mathrm{e}+04$ & $1.4 \mathrm{e}+05$ & 22.12 \\
\hline Median & 6.0 & 0.65 & 16.17 & 4.37 & 1.17 & 0.15 & $1.0 \mathrm{e}+03$ & $1.3 e+03$ & 1.60 \\
\hline Mean & 7.1 & 0.90 & 16.22 & 5.90 & 3.48 & 0.22 & $3.4 \mathrm{e}+03$ & $6.8 \mathrm{e}+03$ & 2.54 \\
\hline Min & 0.7 & 0.07 & 11.88 & 0.71 & 0.05 & 0.03 & $6.3 e+00$ & $3.5 \mathrm{e}+01$ & 0.21 \\
\hline Max & 22.0 & 3.05 & 47.09 & 342.80 & 237.24 & 31.34 & $2.9 \mathrm{e}+05$ & $2.9 \mathrm{e}+06$ & 400.20 \\
\hline Median & 8.1 & 0.67 & 20.31 & 5.27 & 2.08 & 0.25 & $1.6 \mathrm{e}+03$ & $8.7 \mathrm{e}+03$ & 5.31 \\
\hline Mean & 8.0 & 0.75 & 20.81 & 9.68 & 5.36 & 0.49 & $5.0 \mathrm{e}+03$ & $3.6 \mathrm{e}+04$ & 9.48 \\
\hline \multicolumn{10}{|c|}{ Clumps Associated with H II Regions } \\
\hline
\end{tabular}
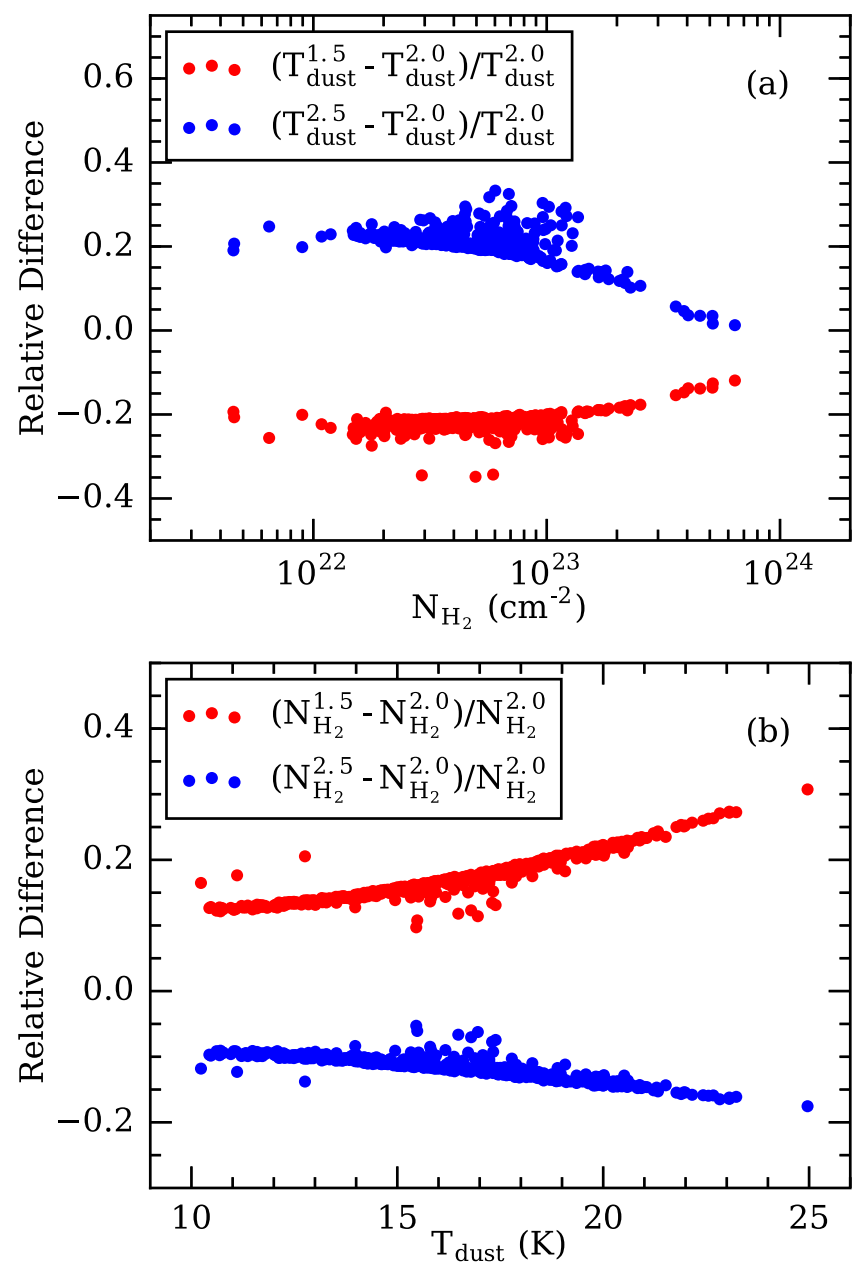

Figure 7. Relative differences of column densities and dust temperatures when using different dust emissivity indices (red symbols for $\beta=1.5$ and blue ones for $\beta=2.5$ ).

where $\kappa_{\nu}(600 \mathrm{GHz})=3.33 \mathrm{~cm}^{2} \mathrm{~g}^{-1}$ is the dust opacity for coagulated grains with thin ice mantles (from column (5) of Table 1 in Ossenkopf \& Henning 1994; often referred to as
OH5), but scaled down by a factor of 1.5 as suggested in Kauffmann et al. (2010). The scaled OH5 dust opacities are consistent with the values used in other high-mass star formation studies (e.g., Kauffmann et al. 2008; Elia et al. 2010, 2013; Kauffmann \& Pillai 2010; Veneziani et al. 2013; Traficante et al. 2015). The dust emissivity index has been fixed to be $\beta=2.0$, in agreement with the standard value for cold dust emission (Hildebrand 1983). The free parameters in this model are the dust temperature and the column density.

The fitting was performed using the Levenberg-Marquardt algorithm provided in the python package $\operatorname{lmfit}{ }^{18}$ (Newville et al. 2016). Only pixels with positive intensities in the four longest-wavelength Hi-GAL bands and the ATLASGAL band were modeled, and the inverses of the rms errors in the images were used as weights in the fit. We found that pixels with $60 \mu \mathrm{m}$ intensities $<60 \mathrm{MJy} \mathrm{sr}^{-1}$ (about $3 \sigma$ ) cannot be well fitted. For these pixels, only the data at wavelengths greater than or equal to $250 \mu \mathrm{m}$ were used.

The resultant column density and dust temperature maps are presented in Figure 6. Most HMSC candidates show coincidence between density maximum and temperature minimum, as is seen in the sources presented in Figure 6. This is in line with the absence of detectable star-forming activity.

\subsection{Physical Parameters}

The beam-averaged column densities and dust temperatures at the peak positions were extracted from the relevant maps and are listed in Table 3.

We integrated the column densities in the scaled ellipses at a resolution of 36!" 4 to estimate the clump mass using the relationship

$$
M_{\text {clump }}=\mu_{\mathrm{H}_{2}} m_{\mathrm{H}} d^{2} \Omega_{\text {pix }} \sum N_{\mathrm{H}_{2}} .
$$

Here $d$ is the source distance and $\Omega_{\text {pix }}$ is the solid angle of one pixel. The major and minor axes of the scaled ellipses were obtained via $\Theta_{36.4}^{\mathrm{maj}}=\sqrt{\Theta_{\mathrm{atl}}^{\mathrm{maj}}-19 ! 2^{2}+36 ! 4^{2}}$ and $\Theta_{36.4}^{\min }=$

$\overline{18 \text { https://lmfit.github.io/lmfit-py/index.html }}$ 

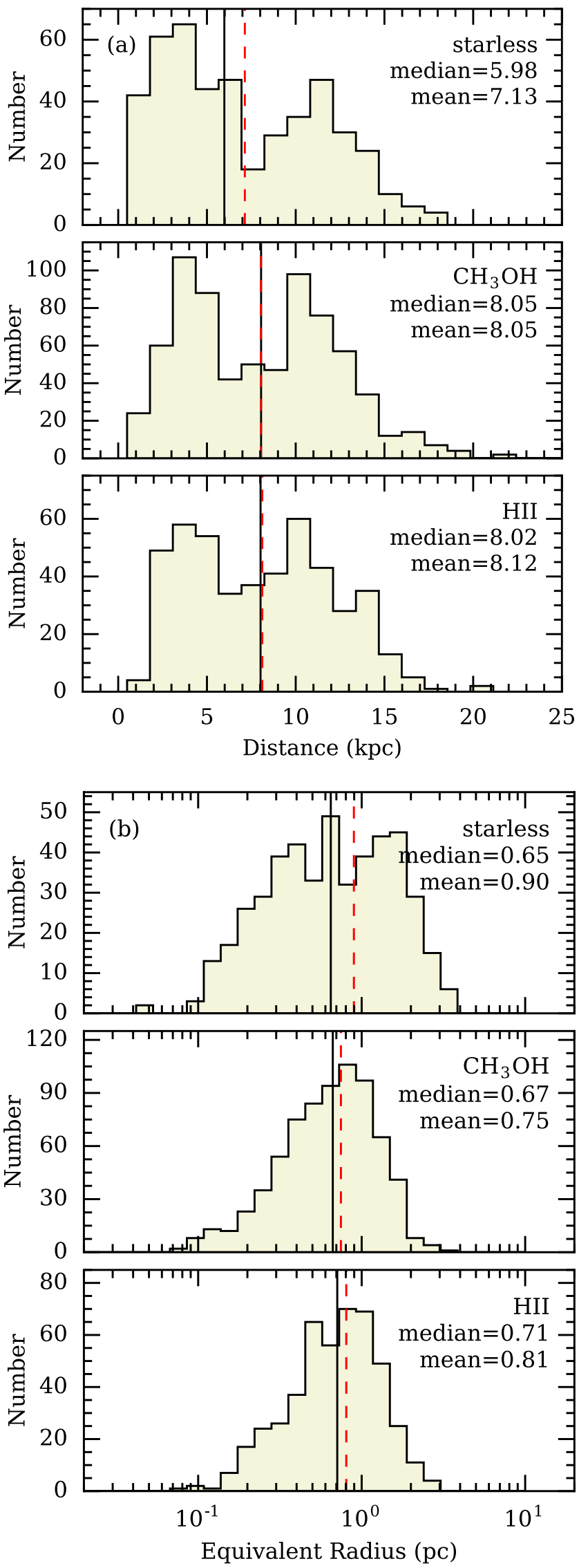

Figure 8. Histograms of distance (a) and equivalent radius (b) of HMSC candidates, clumps associated with methanol masers, and $\mathrm{H}$ II regions. The black solid and red dashed vertical lines mark the median and mean values, respectively.
$\sqrt{\Theta_{\text {atl }}^{\min 2}-19 ! 2^{2}+36 . ! 4^{2}}$, where $\Theta_{\text {atl }}^{\text {maj }}$ and $\Theta_{\text {atl }}^{\min }$ are the major and minor axes given in the ATLASGAL catalog, respectively. The source average $\mathrm{H}_{2}$ number densities were calculated to be

$$
n_{\mathrm{H}_{2}}=\frac{M_{\text {clump }}}{(4 / 3) \pi r_{\mathrm{eq}}^{3} \mu_{\mathrm{H}_{2}} m_{\mathrm{H}}} \text {. }
$$

The mass surface densities were derived via

$$
\Sigma_{\text {mass }}=\frac{M_{\text {clump }}}{\pi r_{\mathrm{eq}}^{2}} .
$$

Here $r_{\mathrm{eq}}$ is the equivalent physical radius.

In the process of fitting the data, we determined the frequencyintegrated intensity $\left(I_{\text {int }}\right)$ for each pixel using the resultant dust temperature and column density. The luminosities of the sources with distance measurements were calculated by integrating the frequency-integrated intensities within the scaled ellipses,

$$
L_{\text {clump }}=4 \pi d^{2} \Omega_{\text {pix }} \sum I_{\text {int }} .
$$

The resulting dust temperatures, column densities, number densities, mass surface densities, masses, and luminosities are given in columns (3)-(8) of Table 3, and the statistics are presented in Table 4. The candidate HMSCs are, in general, cold and dense, with a median temperature of $16 \mathrm{~K}$ and a median column density of $4.4 \times 10^{22} \mathrm{~cm}^{-2}$. The masses range between 8 and $6.1 \times 10^{4} M_{\odot}$, with a median value of 1019 $M_{\odot}$ and a mean of $3384 M_{\odot}$. The luminosities vary from 9 to $1.4 \times 10^{5} L_{\odot}$, with the mean and median luminosities being 1329 and $6838 L_{\odot}$, respectively.

The uncertainties of the parameters largely originate from the uncertainties in distance and dust properties. The typical distance uncertainty is about $10 \%$ and can propagate to other parameters. The preciseness of many parameters would heavily depend on the dust opacity, which is subject to a factor of 2 uncertainty (Ossenkopf \& Henning 1994). The dust emissivity index can also largely influence the parameters from SED fits. Shown in Figure 7 are the relative changes of column densities and dust temperatures at the peak positions of all of the HMSCs when using different dust emissivity indices. The dust temperatures would increase by $13 \%-28 \%$ if an index of 1.5 is adopted and would decrease by 5\%-18\% if 2.5 is used. An index of 1.5 would lead the column densities to decrease by $10 \%-35 \%$, while an index of 2.5 can enlarge the column densities by $2 \%-35 \%$.

\section{Discussion}

In order to investigate whether the HMSC candidates genuinely represent an early phase of high-mass star formation, it is desirable to compare their properties with those of other samples of young high-mass star formation regions. We have obtained dust properties for 728 clumps associated with 6.7 GHz Class II methanol masers (methanol clumps, hereafter) and $469 \mathrm{H}$ II regions ( $\mathrm{H}$ II clumps, hereafter) following the procedure given in Section 5. The methanol clumps were selected based on a spatial cross-match between ATLASGAL sources and $6.7 \mathrm{GHz}$ methanol masers from the MMB survey (Caswell et al. 2010, 2011; Green et al. 2010, 2012; Breen et al. 2015). Only sources residing in the inner Galactic plane were included in the sample. The sources associated with $\mathrm{H}$ II regions were taken from Urquhart et al. (2014c). Similarly, only sources with $|l|<60^{\circ}$ and $|b|<1^{\circ}$ were considered. To 

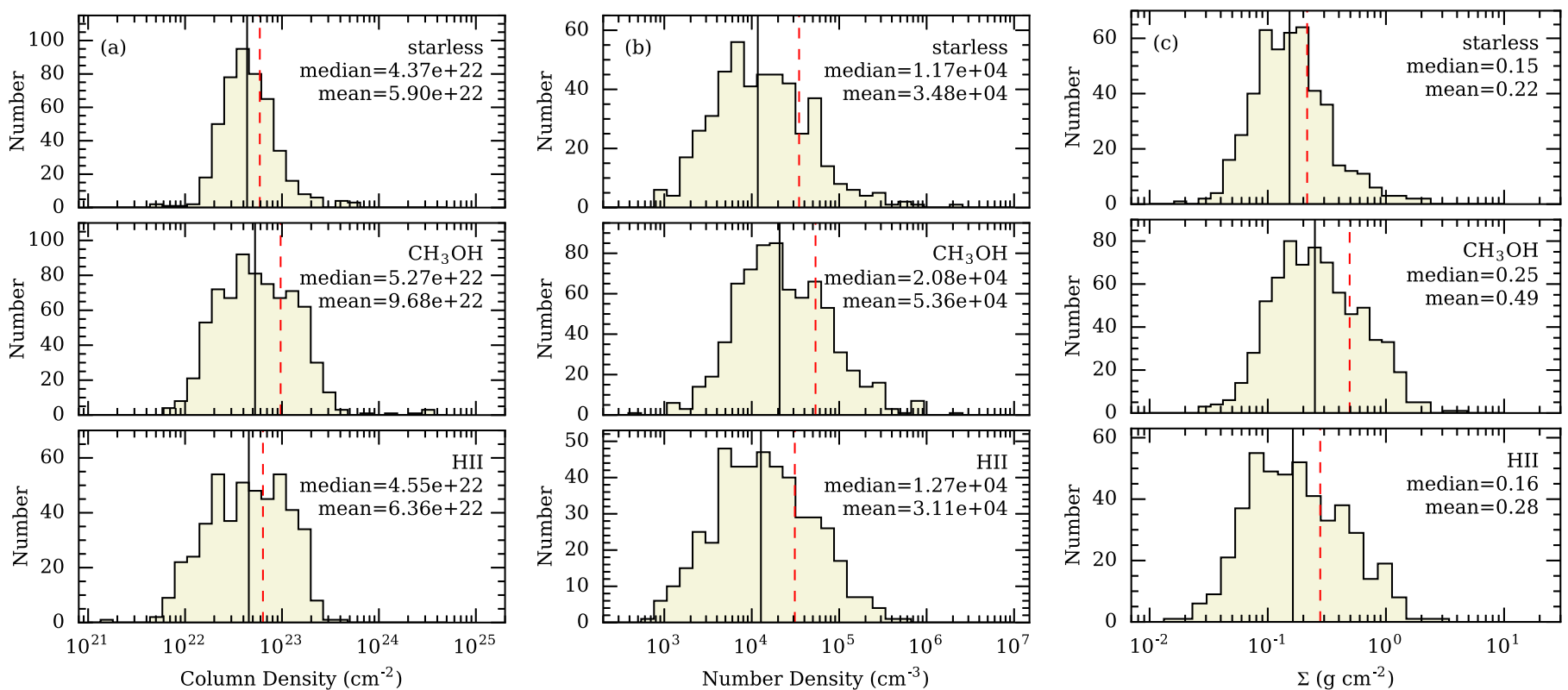

Figure 9. Histograms of $\mathrm{H}_{2}$ column density (a), $\mathrm{H}_{2}$ number density (b), and mass surface density (c) of HMSC candidates, clumps associated with methanol masers, and $\mathrm{H}$ II regions. The black solid and red dashed vertical lines mark the median and mean values, respectively.
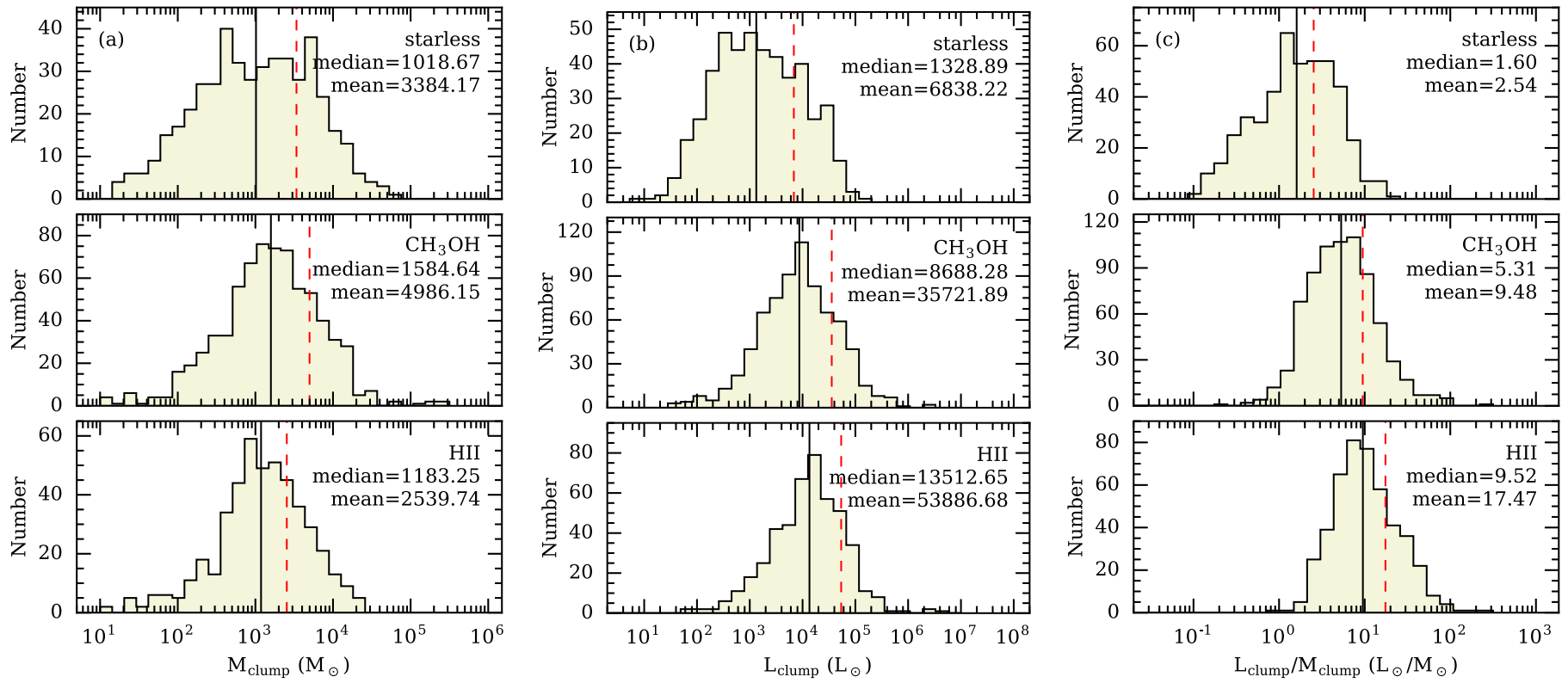

Figure 10. Histograms of clump mass (a), clump luminosity (b), and luminosity-to-mass ratio (c) of HMSC candidates, clumps associated with methanol masers, and $\mathrm{H}$ II regions. The black solid and red dashed vertical lines mark the median and mean values, respectively.

maintain consistency with the HMSC candidate sample, we only included sources with peak intensities $>0.5 \mathrm{Jy}^{\text {beam }}{ }^{-1}$ at $870 \mu \mathrm{m}$. In the following sections, we show that the HMSC candidates are entities similar to the clumps associated with methanol maser and $\mathrm{H}$ II region clumps, which are known to have formed high-mass stars, but the HMSC clumps are at an earlier evolutionary stage.

\subsection{Comparison of Clump Properties}

The starless, methanol, and H II samples have 463 (100\%), 722 (99\%), and 464 (99\%) sources that have systemic velocity information, enabling us to obtain distance measurements using the parallax-based distance estimator (see Section 4).
Histograms of the distances for the three groups of sources are shown in Figure 8(a). The methanol group has a similar median and mean distance to the $\mathrm{H}$ II group, while starless clumps tend to be nearer, with smaller median and mean distances. The fraction of sources at distances farther than $8 \mathrm{kpc}$ is $41 \%, 50 \%$, and $50 \%$ for the starless, methanol, and $\mathrm{H}$ II clumps, respectively. The greater mean distance in the star-forming groups is consistent with the fact that the more evolved clumps tend to show stronger emission at $870 \mu \mathrm{m}$ (He et al. 2016), making the detection of methanol and H II clumps at the far distance easier than for the starless sample.

The difference in the distance distribution of the samples raises the question as to whether they are truly comparable 


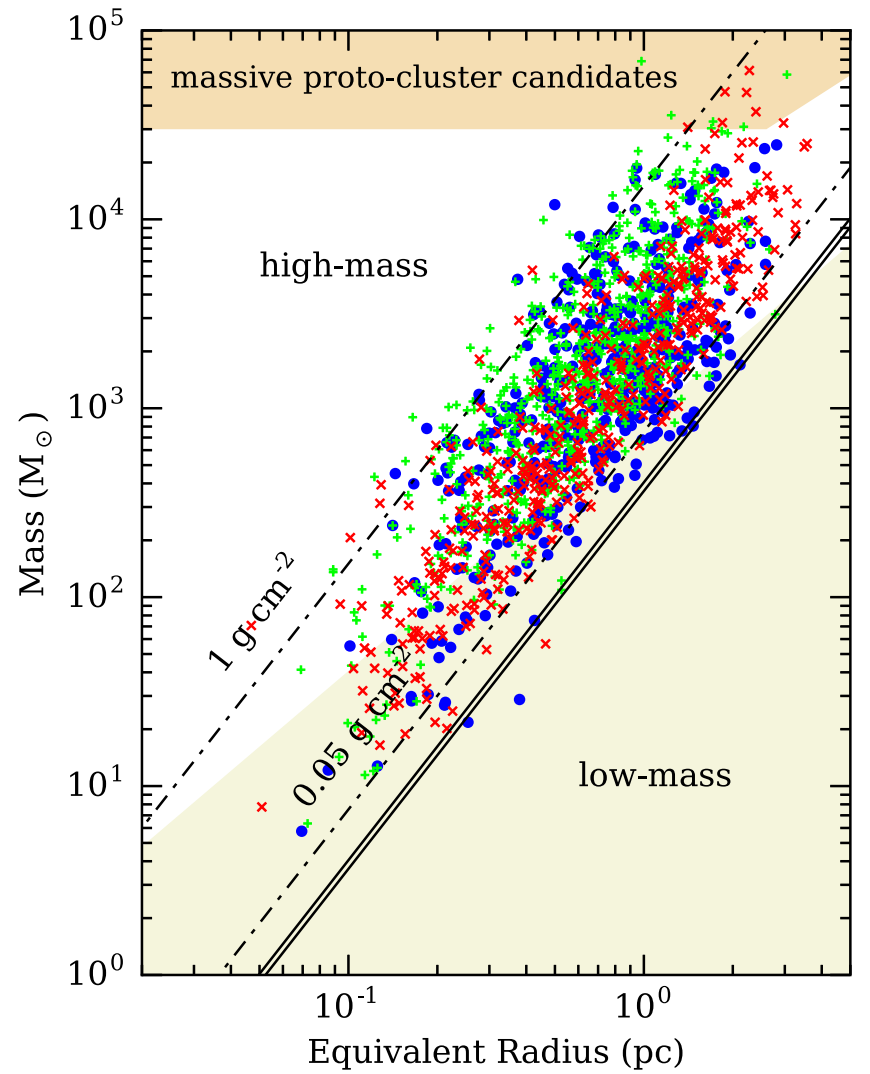

Figure 11. Clump mass as a function of equivalent radius for HMSC candidates (red crosses), clumps associated with methanol masers (green plus signs), and $\mathrm{H}$ II regions (blue filled circles). The unshaded area delimits the region of highmass star formation regions. The threshold, $M>870 M_{\odot}(r / \mathrm{pc})^{1.33}$, is adopted from Kauffmann \& Pillai (2010). The mass surface density thresholds for "efficient star formation" of $116 M_{\odot} \mathrm{pc}^{-2}\left(\sim 0.024 \mathrm{~g} \mathrm{~cm}^{-2}\right)$ from Lada et al. (2010) and $129 M_{\odot} \mathrm{pc}^{-2}\left(\sim 0.027 \mathrm{~g} \mathrm{~cm}^{-2}\right)$ from Heiderman et al. (2010) are shown as black solid lines. The upper and lower dot-dashed lines give two mass surface density cuts of 0.05 and $1 \mathrm{~g} \mathrm{~cm}^{-2}$. The upper shaded region indicates the parameter space for massive protoclusters, defined in Bressert et al. (2012).

groups or if they represent overlapping but different populations. Figures 8(b), 9(a)-(c), and 10(a) show that the three samples have very similar distributions of sizes, densities, and masses, suggesting that they are comparable groups, but at different stages of the star formation process (see Section 6.3). In contrast, increasing densities and masses from starless to $\mathrm{H}$ II clumps have been reported by $\mathrm{He}$ et al. (2016) and Svoboda et al. (2016). Compared to the clumps investigated in He et al. (2016) and Svoboda et al. (2016), those investigated in this work are in general higher mass, as we set a minimum $870 \mu \mathrm{m}$ peak intensity of $0.5 \mathrm{Jy}_{\text {beam }}{ }^{-1}$. We suggest that the inclusion of some lower-mass sources in $\mathrm{He}$ et al. (2016) and Svoboda et al. (2016), especially for objects at early stages, is the main reason that they observe increasing masses and densities from starless to $\mathrm{H}$ II clumps.

\subsection{High-mass Star Birth Sites}

To establish whether we have assembled a robust sample of HMSC candidates, it is crucial to assess their potential to form high-mass stars. Shown in Figure 11 is the mass-radius diagram on which the starless, methanol, and $\mathrm{H}$ II clumps are presented as red crosses, green plus signs, and filled blue circles, respectively. Also overplotted are several thresholds
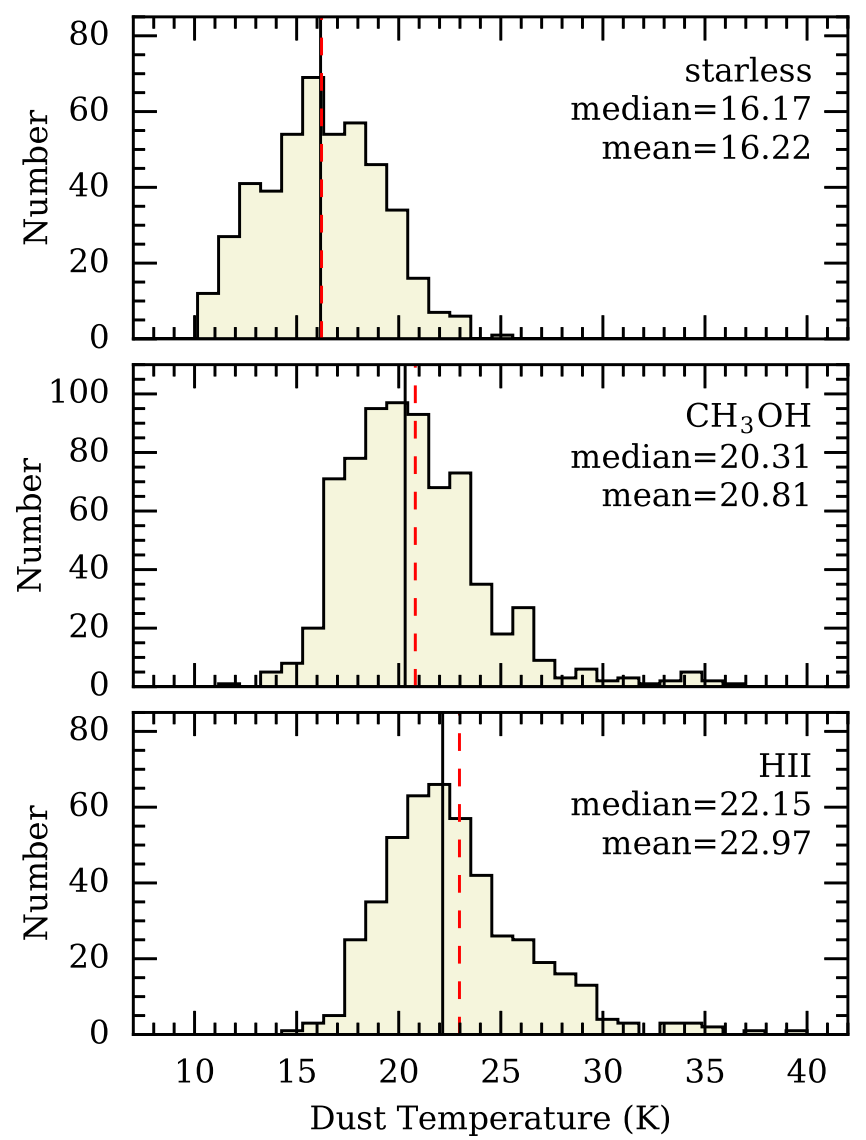

Figure 12. Histograms of dust temperature of HMSC candidates (a), clumps associated with methanol masers (b), and $\mathrm{H}$ II regions (c). The black solid and red dashed vertical lines mark the median and mean values, respectively.

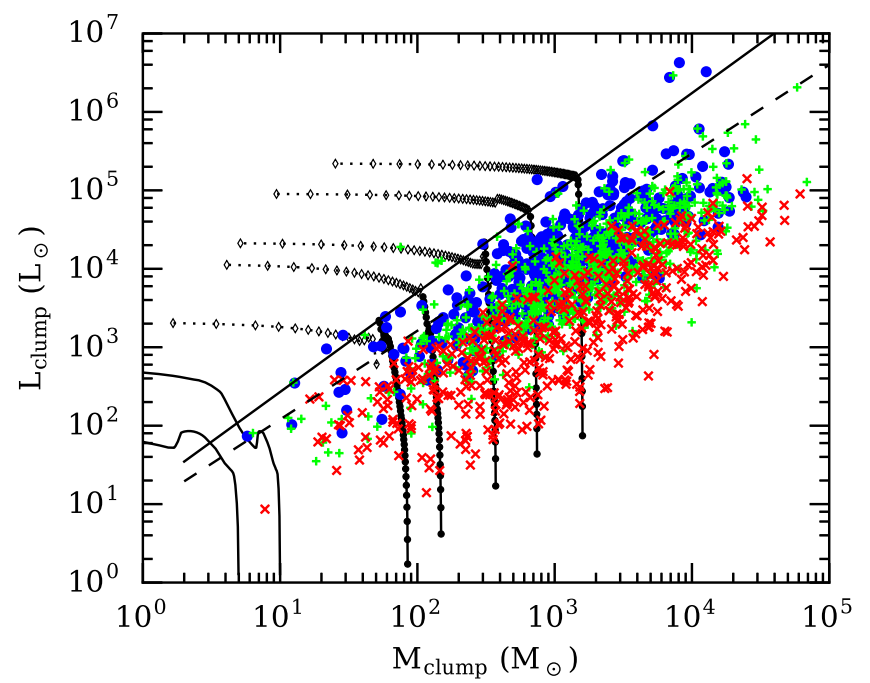

Figure 13. Luminosity-mass diagram for HMSC candidates (red crosses), clumps associated with methanol masers (green plus signs), and $\mathrm{H}$ II regions (blue filled circles). Evolution tracks for stars with final masses of 2.0, 4.0, 6.5, 8.0, 13.5, 18.0, and $35.0 M_{\odot}$ are from Saraceno et al. (1996, solid tracks) and Molinari et al. (2008, tracks with symbols). The solid and dashed lines are the best $\log -\log$ fit for Class I and Class 0 sources, respectively, extrapolated in the high-mass regime by Molinari et al. (2008).

for star formation determined from local clouds. All of the sources with the exception of one starless, three methanol, and three H II clumps meet the criteria for "efficient" star formation of $116 M_{\odot} \mathrm{pc}^{-2}\left(\sim 0.024 \mathrm{~g} \mathrm{~cm}^{-2}\right)$ and $129 M_{\odot} \mathrm{pc}^{-2}$ 


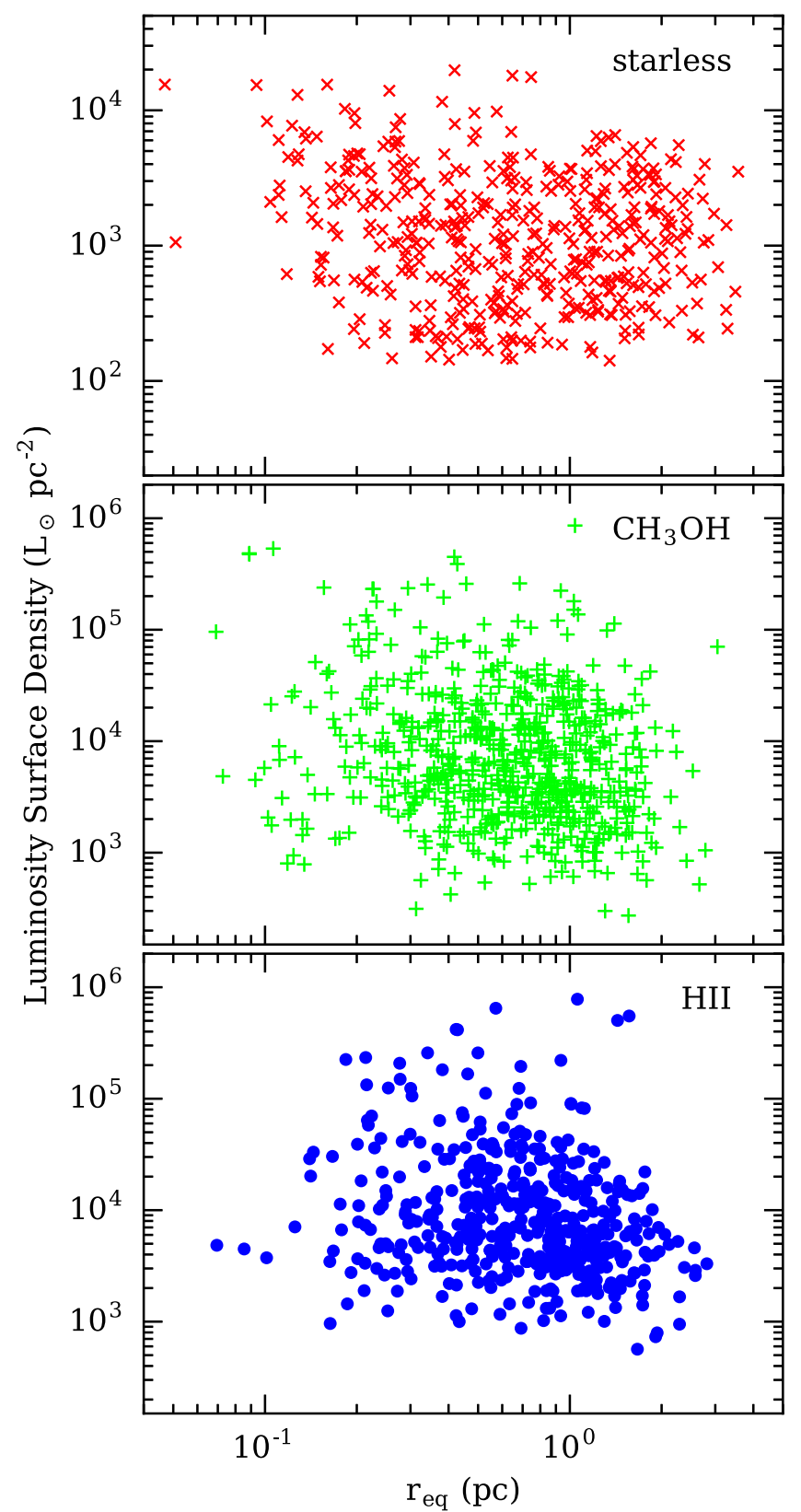

Figure 14. Luminosity surface density vs. equivalent radius for HMSC candidates (top), clumps associated with methanol masers (middle), and $\mathrm{H} \mathrm{II}$ regions (bottom). Note that the ordinate in the top panel covers lower values than in the two lower panels.

$\left(\sim 0.027 \mathrm{~g} \mathrm{~cm}^{-2}\right.$; solid lines) from Lada et al. (2010) and Heiderman et al. (2010).

A more restrictive high-mass star formation criterion of $M \geqslant 870 M_{\odot}\left(R_{\text {eq }} / \mathrm{pc}\right)^{1.33}$ was suggested by Kauffmann \& Pillai (2010) from observations of nearby clouds. About $82 \%$ (378/463) of HMSC candidates are above this threshold and are potential high-mass star-forming regions. The fractions for the methanol and $\mathrm{H}$ II groups fulfilling this threshold are $90 \%$ and $80 \%$, respectively.

Mass surface density, $\Sigma_{\text {mass }}$, is another commonly used parameter to assess the high-mass star formation potential. Krumholz \& McKee (2008) suggest that a minimum mass surface density of $1 \mathrm{~g} \mathrm{~cm}^{-2}$ is required to prevent fragmentation into low-mass cores through radiative feedback, thus allowing

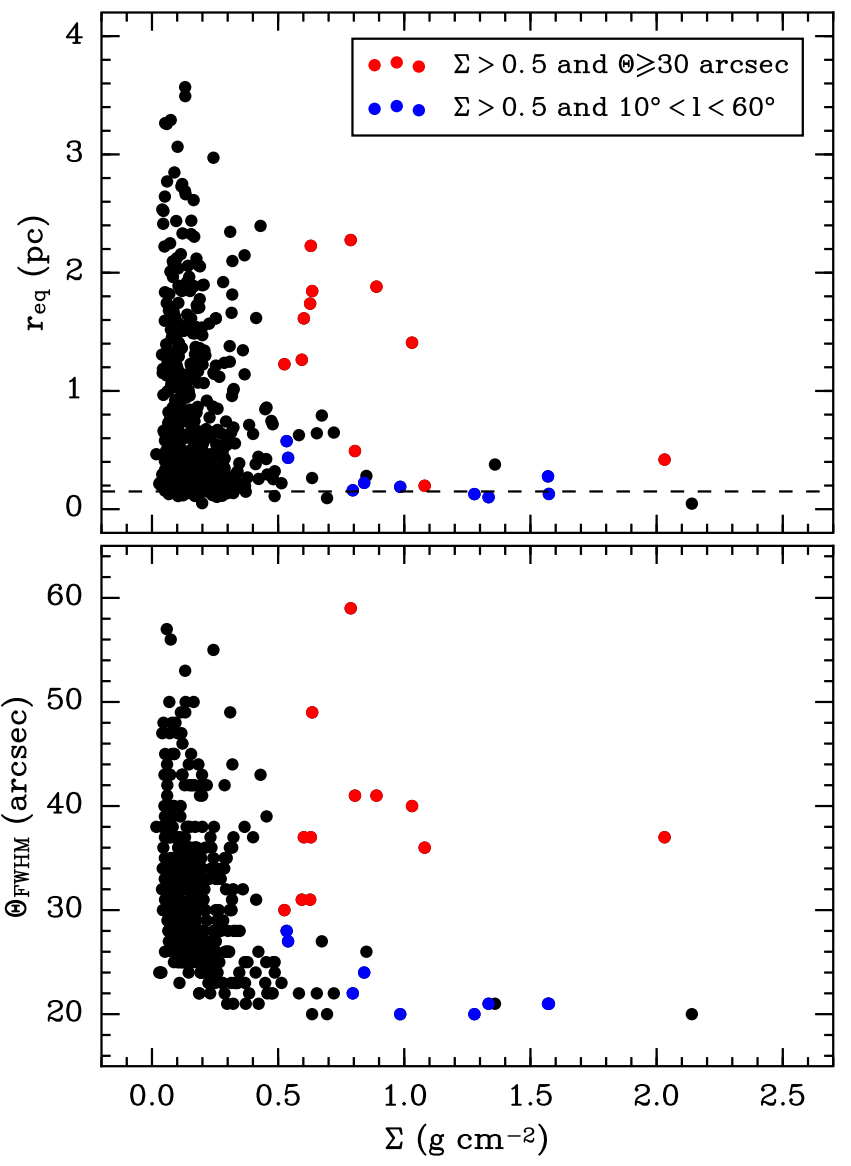

Figure 15. Equivalent radius (top) and angular size (bottom) vs. mass surface density. The sources with mass surface densities $>0.5 \mathrm{~g} \mathrm{~cm}^{-2}$ and angular sizes $>30^{\prime \prime}$ are shown in red, and the ones located in $10^{\circ}<l<60^{\circ}$ are shown in blue. The dashed line in the top panel marks a radius threshold of $0.15 \mathrm{pc}$ for separating cores and clumps.

high-mass star formation. However, this threshold is relatively uncertain, and magnetic fields, which can help prevent fragmentation, were not considered in the calculations. Highmass clumps and cores with $0.05 \leqslant \Sigma \leqslant 0.5 \mathrm{~g} \mathrm{~cm}^{-2}$ are indeed reported in the literature (Butler \& Tan 2012; Peretto et al. 2013; Tan et al. 2013). In a recent study based on ATLASGAL clumps, Urquhart et al. (2014c) suggested a less stringent empirical threshold of $0.05 \mathrm{~g} \mathrm{~cm}^{-2}$. If we adopt a threshold of $1 \mathrm{~g} \mathrm{~cm}^{-2}$, the fraction of high-mass star-forming clump candidates that exceed this is less than $10 \%$, even for the methanol and $\mathrm{H}$ II groups, where high-mass star formation is known to be occurring. In contrast, more than $90 \%$ of clumps exceed the less stringent $0.05 \mathrm{~g} \mathrm{~cm}^{-2}$ threshold. Thus, it appears that the Urquhart et al. (2014c) threshold is more useful for observations that average over a clump. Among the HMSC candidates with distances, there are 448 clumps $(\sim 97 \%)$ fulfilling this threshold and hence having the potential to form high-mass stars.

\subsection{The Very Early Phases of Star Formation}

As a clump evolves from a quiescent phase to one with active star formation, radiative heating from star formation is expected to raise the dust temperature. As $\mathrm{H}$ II regions are more evolved indicators of star formation than methanol masers, H II clumps should be subject to stronger radiative 
Table 5

Physical Parameters for Starless Core Candidates

\begin{tabular}{|c|c|c|c|c|c|c|c|c|c|}
\hline Designation & $\begin{array}{c}\text { Distance } \\
(\mathrm{kpc})\end{array}$ & $\begin{array}{c}r_{\mathrm{eq}} \\
(\mathrm{pc})\end{array}$ & $\begin{array}{c}T_{\text {dust }} \\
(\mathrm{K})\end{array}$ & $\begin{array}{c}N_{\mathrm{H}_{2}} \\
\left(10^{22} \mathrm{~cm}^{-2}\right)\end{array}$ & $\begin{array}{c}n_{\mathrm{H}_{2}} \\
\left(10^{4} \mathrm{~cm}^{-3}\right)\end{array}$ & $\begin{array}{c}\Sigma_{\text {mass }} \\
\left(\mathrm{g} \mathrm{cm}^{-2}\right)\end{array}$ & $\begin{array}{c}M_{\mathrm{cl}} \\
\left(M_{\odot}\right)\end{array}$ & $\begin{array}{c}L_{\mathrm{cl}} \\
\left(L_{\odot}\right)\end{array}$ & $\begin{array}{c}L_{\mathrm{cl}} / M_{\mathrm{cl}} \\
\left(L_{\odot} / M_{\odot}\right)\end{array}$ \\
\hline G000.5184-0.6127 & 3.3 & 0.13 & 18.86 & 1.59 & 11.46 & 0.30 & 81.32 & 392.85 & 4.83 \\
\hline G004.4076+0.0993 & 2.9 & 0.14 & 18.13 & 2.08 & 12.26 & 0.32 & 89.93 & 359.99 & 4.00 \\
\hline G010.2144-0.3051 & 3.1 & 0.13 & 16.64 & 6.58 & 51.83 & 1.28 & 313.76 & 666.97 & 2.13 \\
\hline G014.4876-0.1274 & 3.1 & 0.13 & 13.84 & 6.69 & 63.20 & 1.57 & 393.71 & 247.41 & 0.63 \\
\hline G015.2169-0.4267 & 1.9 & 0.11 & 15.44 & 2.74 & 12.72 & 0.28 & 53.76 & 65.55 & 1.22 \\
\hline G035.2006-0.7253 & 2.2 & 0.10 & 15.22 & 7.67 & 68.13 & 1.33 & 206.63 & 267.73 & 1.30 \\
\hline G327.2585-0.6051 & 2.9 & 0.15 & 18.52 & 2.63 & 13.03 & 0.37 & 122.21 & 439.37 & 3.60 \\
\hline G328.2075-0.5865 & 2.7 & 0.09 & 18.81 & 2.34 & 38.33 & 0.69 & 91.82 & 424.89 & 4.63 \\
\hline G350.7947+0.9075 & 1.4 & 0.12 & 15.01 & 2.62 & 5.43 & 0.12 & 25.80 & 26.87 & 1.04 \\
\hline $\mathrm{G} 350.8162+0.5146$ & 1.3 & 0.15 & 18.20 & 3.05 & 2.89 & 0.08 & 27.46 & 100.12 & 3.65 \\
\hline G351.1414+0.7764 & 1.4 & 0.12 & 18.82 & 4.10 & 10.42 & 0.24 & 51.15 & 201.26 & 3.93 \\
\hline $\mathrm{G} 351.1510+0.7656$ & 1.4 & 0.14 & 18.59 & 3.08 & 5.44 & 0.14 & 39.65 & 146.38 & 3.69 \\
\hline $\mathrm{G} 351.1588+0.7490$ & 1.4 & 0.12 & 21.30 & 3.64 & 7.84 & 0.19 & 42.00 & 364.07 & 8.67 \\
\hline $\mathrm{G} 351.4981+0.6634$ & 1.4 & 0.05 & 16.19 & 6.90 & 236.42 & 2.14 & 70.79 & 107.03 & 1.51 \\
\hline G351.5089+0.6415 & 1.3 & 0.10 & 16.40 & 4.16 & 12.83 & 0.26 & 41.91 & 71.50 & 1.71 \\
\hline G351.5290+0.6939 & 1.3 & 0.11 & 15.18 & 8.54 & 22.72 & 0.49 & 89.91 & 91.47 & 1.02 \\
\hline G351.5663+0.6068 & 1.3 & 0.15 & 13.69 & 5.37 & 7.51 & 0.22 & 73.17 & 41.42 & 0.57 \\
\hline G352.9722+0.9249 & 1.4 & 0.11 & 17.82 & 3.60 & 7.90 & 0.17 & 31.95 & 109.04 & 3.41 \\
\hline G353.0114+0.9828 & 1.3 & 0.14 & 18.93 & 2.24 & 3.58 & 0.10 & 31.05 & 135.10 & 4.35 \\
\hline G353.0195+0.9750 & 1.3 & 0.14 & 18.44 & 3.06 & 3.17 & 0.09 & 26.60 & 102.84 & 3.87 \\
\hline
\end{tabular}

heating. Thus, dust temperature, in general, should serve as a tracer of evolutionary stage (Mueller et al. 2002). Figure 12 shows the distribution of dust temperatures for the three groups. The median values are 16.2, 20.3, and 22.2 for starless, methanol, and H II clumps, respectively. This trend of increasing dust temperature is consistent with an evolutionary sequence from starless to $\mathrm{H}$ II clumps. A K-S test shows that the probability for the three distributions to be the same is smaller than $0.1 \%$, indicating that the HMSC candidates represent an earlier phase of star formation.

The distributions of luminosities for the three samples are presented in Figure 10(b), where again an increasing trend can be observed. The median luminosities are 1329,8688 , and $13,513 L_{\odot}$ for starless, methanol, and H II clumps, respectively. We attribute this difference in luminosity to emission arising from warm cores with embedded protostars. In starless clumps, emission from dust envelopes, heated externally, dominates the luminosity. In contrast, warmer cores in methanol and $\mathrm{H}$ II clumps significantly contribute to the luminosities. Since there can be significant emission at wavelengths shorter than $70 \mu \mathrm{m}$, the genuine luminosities for clumps with embedded warm cores will be even higher than the values estimated in this work.

Another effective tool for diagnosing different stages of dense structures in molecular clouds is the luminosity-mass $\left(L_{\text {clump }}-M_{\text {clump }}\right)$ diagram, on which sources at different phases of star formation can be readily distinguished (Molinari et al. 2008, 2016a). This diagram for high-mass star formation was introduced by Molinari et al. (2008) based on the twophase model of McKee \& Tan (2003). In the first phase, the mass of a core slightly decreases owing to accretion and molecular outflows, while the luminosity increases significantly, and the source follows an almost vertical track in the $L_{\text {clump }}-M_{\text {clump }}$ diagram. In the second phase, the surrounding material is expelled through radiation and molecular outflows. With a nearly constant luminosity, the object follows an almost horizontal path. Although the evolutionary tracks have been initially modeled for single cores, the $L_{\text {clump }}-M_{\text {clump }}$ diagram has been also frequently used to discuss the evolution of clumps (e.g., Elia et al. 2010, 2013; Traficante et al. 2015; Wyrowski et al. 2016)

The $L_{\text {clump }}-M_{\text {clump }}$ diagram is shown in Figure 13 with the same symbol convention as that in Figure 11. Also overplotted are the theoretical evolutionary tracks for the low- and highmass regimes adopted from Molinari et al. (2008). The best $\log -\log$ fits for Class I and Class 0 sources extrapolated in the high-mass regime by Molinari et al. (2008) are shown as solid and dashed lines, respectively. Although there is a degree of overlap, segregated parameter spaces are occupied by different groups of clumps. With a median luminosity-to-mass ratio ( $L_{\text {clump }} / M_{\text {clump }}$ ) of about $1.6 L_{\odot} / M_{\odot}$, the HMSC candidates are mainly located toward the bottom right of the diagram. The $L_{\text {clump }} / M_{\text {clump }}$ values we find are comparable to those of starless clumps reported in Traficante et al. (2015) and significantly lower than those of known protostellar clumps (e.g., Urquhart et al. 2014c; Traficante et al. 2015).

Molinari et al. (2016a) suggest that $L_{\text {clump }} / M_{\text {clump }}<1 L_{\odot} / M_{\odot}$ is characteristic of starless clumps; however, some of our HMSC candidates have a larger luminosity-to-mass ratio. A possible reason for many HMSC candidates having $L M$ ratios higher than the suggested threshold of $1 L_{\odot} / M_{\odot}$ is that some may be externally heated by the interstellar radiation field (ISRF). For a source externally heated by ISRF, the luminosity surface density would keep constant. In contrast, the luminosity surface density for a star-forming clump will decrease with increasing radius because of the internal heating from embedded protostars, which dominate the observed luminosities for smaller sources. Plots of luminosity surface density versus equivalent radius for the three samples are shown in Figure 14. Note that the ordinate in the top panel covers lower values than in the two lower panels. A weak decreasing trend can be seen for methanol and H II clumps, while the HMSC candidates are scattered in luminosity surface density versus radius parameter space. This is consistent with the methanol and $\mathrm{H}$ II clumps being internally heated while the starless clumps are externally heated by the ISRF. If this is the case, one would expect smaller $L / M$ ratios for starless clumps if 
the masses and luminosities are measured over smaller regions. When we calculate the luminosities and masses in just one beam, the median, mean, and maximum $L / M$ ratios for the HMSC sample decrease from $1.60,2.54$, and 22.12 to $1.36,2.19$, and 20.59 , respectively. In contrast, the $L / M$ ratios increase by about $11 \%$ and $30 \%$ for methanol and $\mathrm{H}$ II clumps, respectively. This provides further evidence of external heating for the HMSC candidates and internal heating for the (methanol and $\mathrm{H}$ II) starforming clumps.

\subsection{Comparison with the BGPS Starless Clumps}

Our results differ substantially from those of Svoboda et al. (2016). Our sample has higher median masses, surface densities, and volume densities. As a result, there is much more overlap between the starless sample and the samples with ongoing star formation in these properties (see Figures 9 and 10) than was found by Svoboda et al. (2016). There are small differences in the assumptions about opacity and the method of determining temperatures, as well as a slightly higher median distance, all of which tend to result in higher masses in our sample, but these are relatively small effects. The primary differences arise from the expanded longitude coverage, the sample inclusion criteria, the spatial resolution, and the source extraction method.

Svoboda et al. (2016) considered sources with longitude exceeding $10^{\circ}$, while our range of $-60^{\circ}<l<60^{\circ}$ includes the sources near the Galactic center and some massive complexes in the fourth quadrant. If considering sources in $10^{\circ}<l<60^{\circ}$ only, the median mass of our HMSC candidates would decrease by $30 \%$, but the median surface and column densities do not change significantly. Svoboda et al. included sources as weak as $100 \mathrm{mJy}$ at $1.1 \mathrm{~mm}$, while we required the flux density at $870 \mu \mathrm{m}$ to be at least $500 \mathrm{mJy}_{\text {beam }}{ }^{-1}$. Consequently, the median masses will be skewed higher in our sample $\left(1019 M_{\odot}\right)$ than in the Svoboda sample $\left(228 M_{\odot}\right)$.

Perhaps the more puzzling difference between the samples is in the surface densities. Our sample has 234 sources with $\Sigma>0.15 \mathrm{~g} \mathrm{~cm}^{-2}$, while Svoboda et al. have only 11 such sources. We examined the nature of these high surface density sources, focusing on the extreme cases with $\Sigma>0.5 \mathrm{~g} \mathrm{~cm}^{-2}$. These fall into two distinctly different categories, as can be seen in Figure 15, where we plot size (angular and linear) versus $\Sigma$. About nine are large $\left(r_{\mathrm{eq}}>1\right.$ pc) clumps with very high $\Sigma>0.5 \mathrm{~g} \mathrm{~cm}^{-2}$, and all lie in the Galactic center region, which was excluded from the Svoboda sample. The others are compact, with angular sizes less than $30^{\prime \prime}$. These lie within larger clumps identified by Svoboda et al. and are favored by the better spatial resolution of our sample, as well as the source finding method (Gaussclump) (Section 2), which seeks small sources within extended structure, versus the method used by Svoboda, which is biased against splitting sources into smaller structures. These small $r_{\mathrm{eq}}<0.15 \mathrm{pc}$ sources are candidates for dense cores, rather than clumps. Some have masses exceeding $100 M_{\odot}$. While follow-up studies will be needed, these are at least candidates for the formation sites of individual, massive stars (see Section 6.5).

\subsection{Possible High-mass Starless Cores}

As shown in Figure 15, some sources have small physical sizes and high mass surface densities and probably represent cores embedded in clumps. Although there is no sharp definition, parsec-scale entities are frequently referred to be clumps that would form a cluster of stars, and smaller structures with sizes of $0.01-0.3 \mathrm{pc}$ are commonly treated as cores that may form one or a group of stars (Bergin \& Tafalla 2007; Zhang et al. 2015, and references therein). If we follow Bergin \& Tafalla (2007) and adopt $0.3 \mathrm{pc}$ as a threshold for discriminating between cores and clumps, about 4.3\% (20/ 463 ) of our HMSC candidates with equivalent radii $<0.15 \mathrm{pc}$ would be high-mass starless core candidates.

The physical parameters of these 20 possible starless cores are given in Table 5. Fifteen of them have distances smaller than $2 \mathrm{kpc}$, and 14 reside in the well-known NGC 6334/6357 star-forming complex (Russeil et al. 2010). Although further deep spectral imaging observations at a high resolution are needed to check the quality of these cores, the large masses $(>26)$ and high densities $\left(\Sigma \geqslant 0.08 \mathrm{~g} \mathrm{~cm}^{-2}\right)$ still make them promising and have the potential to form massive stars.

\section{Summary}

We have utilized data from multiple Galactic surveys to identify hundreds of HMSC candidates distributed throughout the inner Galactic plane. The combination of multiwavelength far-IR and submillimeter continuum data enabled us to obtain some basic parameters, and we have compared these with those of clumps associated with methanol masers and $\mathrm{H}$ II regions. The main findings in this work are summarized as follows:

1. From more than 10,000 dense sources detected in the ATLASGAL survey, a sample of 463 HMSC candidates were identified based on Spitzer/GLIMPSE, Spitzer/ MIPSGAL, Herschel/Hi-GAL, and APEX / ATLASGAL survey data. These clumps are not associated with any known star-forming indicators; the HMSC candidates represent a highly reliable catalog of starless objects.

2. Distances for all 463 HMSC candidates were determined based on their systemic velocities. Their distribution in Galactic longitude is similar to that observed in the whole sample of ATLASGAL clumps, showing overdensities toward the Galactic center and several well-known starforming regions. While plotted on the face-on Milky Way, the majority of the sources are located in spiral arms in the inner Galaxy, with galactocentric distances less than $8.34 \mathrm{kpc}$.

3. Some basic parameters were derived via fitting data at wavelengths from 160 to $870 \mu \mathrm{m}$ to modified blackbodies. These HMSC candidates have a median beamaveraged $\mathrm{H}_{2}$ column density of $4.4 \times 10^{22} \mathrm{~cm}^{-2}$, a median mass of $1019 M_{\odot}$, a median luminosity of 1329 $L_{\odot}$, and a median luminosity-to-mass ratio of $1.6 L_{\odot} / M_{\odot}$.

4. More than 700 clumps associated with $6.7 \mathrm{GHz}$ methanol masers and more than 400 clumps associated with $\mathrm{H}$ II regions were scrutinized using the same analysis techniques to enable us to carry out comparative diagnosis with the newly identified starless clumps. These comparison samples have median size, mass, and density similar to those of high-mass star-forming clumps. The HMSC candidates may be common entities, but they are at an earlier evolutionary stage.

5. All of the HMSC candidates except one fulfill the star formation threshold proposed in Lada et al. (2010) and Heiderman et al. (2010). In the mass-radius diagram, more than $80 \%(378 / 463)$ of starless clumps are above 
the relationship for high-mass star formation proposed by Kauffmann \& Pillai (2010), About 97\% of HMSC candidates have mass surface densities above the threshold defined in Urquhart et al. (2014c), suggesting that most of them have the potential to form high-mass stars.

6. Our HMSC candidates have median and mean dust temperatures of 16.17 and $16.21 \mathrm{~K}$, respectively, significantly colder than star-forming clumps and consistent with other samples of starless clumps reported in the literature. The median luminosity-to-mass ratio of the HMSC candidates is as low as $1.6 L_{\odot} / M_{\odot}$. Further analysis shows that these HMSCs are externally heated, suggesting that these objects truly represent a very early phase of high-mass star formation.

7. Compared to the BGPS starless clumps in Svoboda et al. (2016), our HMSC candidates have larger masses and higher densities, which is mainly due to the inclusion of the $-10^{\circ}<l<10^{\circ}$ region, the higher spatial resolution of ATLASGAL observations, and the different source extraction method.

8. There are 20 HMSC candidates with $r_{\mathrm{eq}}<0.15 \mathrm{pc}$. With small sizes, large masses, and high densities, they may represent a small sample of high-mass starless cores.

We have identified the largest and most reliable HMSC candidates ever reported. Distributed throughout the entire inner Galactic plane, these objects are ideal targets for further investigations on early stages of high-mass star formation. Follow-up observations toward these sources with higher resolution and wider band coverage will contribute to hunting for high-mass starless cores, understanding the fragmentation process, and revealing early chemistry.

We are grateful to an anonymous referee for the constructive comments that helped us improve this paper. This work is supported by the National Natural Science Foundation of China through grants $11503035,11573036,11373009,11433008$, and 11403040, the International S\&T Cooperation Program of China through grant 2010DFA02710, the Beijing Natural Science Foundation through grant 1144015 , the China Ministry of Science and Technology under State Key Development Program for Basic Research through grant 2012CB821800, and the Young Researcher Grant of National Astronomical Observatories, Chinese Academy of Sciences. K.W. is supported by grant WA3628-1/1 through the DFG priority program 1573 "Physics of the Interstellar Medium." We thank Brain Svoboda for helpful discussions about the differences between parameters in Svoboda et al. (2016) and this work.

This research has made use of the NASA/IPAC Infrared Science Archive, which is operated by the Jet Propulsion Laboratory, California Institute of Technology, under contract with the National Aeronautics and Space Administration. This research also has made use of the SIMBAD database, operated at CDS, Strasbourg, France. This work is based in part on observations made with the Spitzer Space Telescope, which is operated by the Jet Propulsion Laboratory, California Institute of Technology, under a contract with NASA. This research made use of APLpy and Astropy for visualization and some analysis. APLpy is an open-source plotting package for Python hosted at http://aplpy.github.com. And Astropy is a community-developed core Python package for Astronomy (Astropy Collaboration et al. 2013).

\section{References}

Aguirre, J. E., Ginsburg, A. G., Dunham, M. K., et al. 2011, ApJS, 192, 4 Astropy Collaboration, Robitaille, T. P., Tollerud, E. J., et al. 2013, A\&A, 558, A33

Barnes, P. J., Muller, E., Indermuehle, B., et al. 2015, ApJ, 812, 6

Benjamin, R. A., Churchwell, E., Babler, B. L., et al. 2003, PASP, 115, 953 Bergin, E. A., \& Tafalla, M. 2007, ARA\&A, 45, 339

Beuther, H., Henning, T., Linz, H., et al. 2015, A\&A, 581, A119

Beuther, H., Linz, H., Tackenberg, J., et al. 2013, A\&A, 553, A115

Bonnell, I. A., Bate, M. R., Clarke, C. J., \& Pringle, J. E. 2001, MNRAS, 323, 785

Breen, S. L., Ellingsen, S. P., Contreras, Y., et al. 2013, MNRAS, 435, 524 Breen, S. L., Fuller, G. A., Caswell, J. L., et al. 2015, MNRAS, 450, 4109 Bressert, E., Ginsburg, A., Bally, J., et al. 2012, ApJL, 758, L28

Butler, M. J., \& Tan, J. C. 2012, ApJ, 754, 5

Carey, S. J., Noriega-Crespo, A., Mizuno, D. R., et al. 2009, PASP, 121, 76

Caswell, J. L., Fuller, G. A., Green, J. A., et al. 2010, MNRAS, 404, 1029 Caswell, J. L., Fuller, G. A., Green, J. A., et al. 2011, MNRAS, 417, 1964 Churchwell, E., Babler, B. L., Meade, M. R., et al. 2009, PASP, 121, 213

Contreras, Y., Schuller, F., Urquhart, J. S., et al. 2013, A\&A, 549, A45 Csengeri, T., Urquhart, J. S., Schuller, F., et al. 2014, A\&A, 565, A75

Cyganowski, C. J., Brogan, C. L., Hunter, T. R., et al. 2014, ApJL, 796, L2 Dempsey, J. T., Thomas, H. S., \& Currie, M. J. 2013, ApJS, 209, 8

Dunham, M. K., Rosolowsky, E., Evans, N. J., II, Cyganowski, C., \& Urquhart, J. S. 2011, ApJ, 741, 110

Elia, D., Molinari, S., Fukui, Y., et al. 2013, ApJ, 772, 45

Elia, D., Schisano, E., Molinari, S., et al. 2010, A\&A, 518, L97

Feng, S., Beuther, H., Zhang, Q., et al. 2016, ApJ, 828, 100

Gallaway, M., Thompson, M. A., Lucas, P. W., et al. 2013, MNRAS, 430, 808

Green, J. A., Caswell, J. L., Fuller, G. A., et al. 2010, MNRAS, 409, 913

Green, J. A., Caswell, J. L., Fuller, G. A., et al. 2012, MNRAS, 420, 3108

Gutermuth, R. A., \& Heyer, M. 2015, AJ, 149, 64

Gutermuth, R. A., Megeath, S. T., Myers, P. C., et al. 2009, ApJS, 184, 18

He, Y.-X., Zhou, J.-J., Esimbek, J., et al. 2016, MNRAS, 461, 2288

Heiderman, A., Evans, N. J., II, Allen, L. E., Huard, T., \& Heyer, M. 2010, ApJ, 723, 1019

Hildebrand, R. H. 1983, QJRAS, 24, 267

Jackson, J. M., Finn, S. C., Rathborne, J. M., Chambers, E. T., \& Simon, R. 2008, ApJ, 680, 349

Jackson, J. M., Rathborne, J. M., Foster, J. B., et al. 2013, PASA, 30, 57

Kauffmann, J., Bertoldi, F., Bourke, T. L., Evans, N. J., II, \& Lee, C. W. 2008, A\&A, 487, 993

Kauffmann, J., \& Pillai, T. 2010, ApJL, 723, L7

Kauffmann, J., Pillai, T., Shetty, R., Myers, P. C., \& Goodman, A. A. 2010, ApJ, 712, 1137

Kong, S., Tan, J. C., Caselli, P., et al. 2017, ApJ, 834, 193

Krumholz, M. R., \& McKee, C. F. 2008, Natur, 451, 1082

Lada, C. J., Lombardi, M., \& Alves, J. F. 2010, ApJ, 724, 687

Lumsden, S. L., Hoare, M. G., Urquhart, J. S., et al. 2013, ApJS, 208, 11

McKee, C. F., \& Tan, J. C. 2003, ApJ, 585, 850

Molinari, S., Merello, M., Elia, D., et al. 2016a, ApJL, 826, L8

Molinari, S., Pezzuto, S., Cesaroni, R., et al. 2008, A\&A, 481, 345

Molinari, S., Schisano, E., Elia, D., et al. 2016b, A\&A, 591, A149

Molinari, S., Swinyard, B., Bally, J., et al. 2010, PASP, 122, 314

Mueller, K. E., Shirley, Y. L., Evans, N. J., II, \& Jacobson, H. R. 2002, ApJS, 143,469

Newville, M., Stensitzki, T., Allen, D. B., et al. 2016, Lmfit: Non-Linear LeastSquare Minimization and Curve-Fitting for Python, Astrophysics Source Code Library, ascl:1606.014

Ossenkopf, V., \& Henning, T. 1994, A\&A, 291, 943

Peretto, N., Fuller, G. A., Duarte-Cabral, A., et al. 2013, A\&A, 555, A112 Planck Collaboration, Ade, P. A. R., Aghanim, N., et al. 2014, A\&A, 571, A13 Purcell, C. R., Longmore, S. N., Walsh, A. J., et al. 2012, MNRAS, 426, 1972 Reid, M. J., Dame, T. M., Menten, K. M., \& Brunthaler, A. 2016, ApJ, 823, 77 Russeil, D., Zavagno, A., Motte, F., et al. 2010, A\&A, 515, A55

Saraceno, P., Andre, P., Ceccarelli, C., Griffin, M., \& Molinari, S. 1996, A\&A, 309,827

Sato, M., Reid, M. J., Brunthaler, A., \& Menten, K. M. 2010, ApJ, 720, 1055 Schuller, F., Menten, K. M., Contreras, Y., et al. 2009, A\&A, 504, 415

Shirley, Y. L., Ellsworth-Bowers, T. P., Svoboda, B., et al. 2013, ApJS, 209, 2 Simpson, J. P., Cotera, A. S., Burton, M. G., et al. 2012, MNRAS, 419, 211 Skrutskie, M. F., Cutri, R. M., Stiening, R., et al. 2006, AJ, 131, 1163 Svoboda, B. E., Shirley, Y. L., Battersby, C., et al. 2016, ApJ, 822, 59 Tackenberg, J., Beuther, H., Henning, T., et al. 2012, A\&A, 540, A113 
Tan, J. C., Beltrán, M. T., Caselli, P., et al. 2014, in Protostars and Planets VI, ed. H. Beuther et al. (Tucson, AZ: University of Arizona Press), 27

Tan, J. C., Kong, S., Butler, M. J., Caselli, P., \& Fontani, F. 2013, ApJ, 779, 96

Tan, J. C., Kong, S., Zhang, Y., et al. 2016, ApJL, 821, L3

Traficante, A., Calzoletti, L., Veneziani, M., et al. 2011, MNRAS, 416, 2932

Traficante, A., Fuller, G. A., Peretto, N., Pineda, J. E., \& Molinari, S. 2015, MNRAS, 451, 3089

Urquhart, J. S., Busfield, A. L., Hoare, M. G., et al. 2007, A\&A, 474, 891

Urquhart, J. S., Csengeri, T., Wyrowski, F., et al. 2014a, A\&A, 568, A41

Urquhart, J. S., Figura, C. C., Moore, T. J. T., et al. 2014b, MNRAS, 437, 1791

Urquhart, J. S., Moore, T. J. T., Csengeri, T., et al. 2014c, MNRAS, 443, 1555
Urquhart, J. S., Thompson, M. A., Moore, T. J. T., et al. 2013, MNRAS, 435,400

Vasyunina, T., Linz, H., Henning, T., et al. 2009, A\&A, 499, 149

Veneziani, M., Elia, D., Noriega-Crespo, A., et al. 2013, A\&A, 549, A130

Wang, K., Testi, L., Burkert, A., et al. 2016, ApJS, 226, 9

Wang, K., Zhang, Q., Testi, L., et al. 2014, MNRAS, 439, 3275

Wang, K., Zhang, Q., Wu, Y., \& Zhang, H. 2011, ApJ, 735, 64

Werner, M. W., Roellig, T. L., Low, F. J., et al. 2004, ApJS, 154, 1

Wienen, M., Wyrowski, F., Schuller, F., et al. 2012, A\&A, 544, A146

Wyrowski, F., Güsten, R., Menten, K. M., et al. 2016, A\&A, 585, A149

Zhang, Q., Wang, K., Lu, X., \& Jiménez-Serra, I. 2015, ApJ, 804, 141 